Research Article

\title{
Nonlinear Spring-Mass-Damper Modeling and Parameter Estimation of Train Frontal Crash Using CLGAN Model
}

\author{
Shaodi Dong $\left(\mathbb{D},{ }^{1}\right.$ Zhao Tang $\mathbb{D}^{1},{ }^{1}$ Xiaosong Yang, ${ }^{2}$ Michelle $\mathrm{Wu},{ }^{2}$ Jianjun Zhang, \\ Tao Zhu (D), ${ }^{1}$ and Shoune Xiao ${ }^{1}$ \\ ${ }^{1}$ State Key Laboratory of Traction Power, Southwest Jiaotong University, Chengdu, China \\ ${ }^{2}$ National Centre for Computer Animation, Bournemouth University, Poole, UK \\ Correspondence should be addressed to Zhao Tang; tangzhao@swjtu.edu.cn
}

Received 23 January 2020; Revised 21 June 2020; Accepted 22 July 2020; Published 31 August 2020

Academic Editor: Francisco Beltran-Carbajal

Copyright (C) 2020 Shaodi Dong et al. This is an open access article distributed under the Creative Commons Attribution License, which permits unrestricted use, distribution, and reproduction in any medium, provided the original work is properly cited.

Due to the complexity of a train crash, it is a challenging process to describe and estimate mathematically. Although different mathematical models have been developed, it is still difficult to balance the complexity of models and the accuracy of estimation. This paper proposes a nonlinear spring-mass-damper model of train frontal crash, which achieves high accuracy and maintains low complexity. The Convolutional Long-short-term-memory Generation Adversarial Network (CLGAN) model is applied to study the nonlinear parameters dynamic variation of the key components of a rail vehicle (e.g., the head car, anticlimbing energy absorber, and the coupler buffer devices). Firstly, the nonlinear lumped model of train frontal crash is built, and then the physical parameters are deduced in twenty different cases using D'Alembert's principle. Secondly, the input/output relationship of the CLGAN model is determined, where the inputs are the nonlinear physical parameters in twenty initial conditions, and the output is the nonlinear relationship between the train crash nonlinear parameters under other initial cases. Finally, the train crash dynamic characteristics are accurately estimated during the train crash processes through the training of the CLGAN model, and then the crash processes under different given conditions can be described effectively. The estimation results exhibit good agreement with finite element (FE) simulations and experimental results. Furthermore, the CLGAN model shows great potential in nonlinear estimation, and CLGAN can better describe the variation of nonlinear spring damping compared with the traditional model. The nonlinear spring-mass-damper modeling is involved in improving the speed and accuracy of the train crash estimation, as well as being able to offer guidance for structure optimization in the early design stage.

\section{Introduction}

Among railway accidents, train crashes are considered to be one of the most serious rail disasters, leading to a great number of casualties as well as property losses, as in the case of the railway traffic accident that occurred on the Ningzhou-Wenzhou line in China, resulting in 40 deaths and 172 injuries, and the South Carolina train crash accident, resulting in 2 deaths and 116 injuries. In the past years, researchers and manufacturers have gradually developed new active safety technologies, such as the automatic train operation (ATO) [1], the automatic train adjustment (ATA) [2], the intelligent train operation (ITO) [3], and the train crash detection system (TCD) [4]. However, passive safety systems, in particular crashworthiness design, are still serving as the base for train safety and are therefore widely researched by vehicle engineers and researchers.

Train crash scenarios can be classified into four different modes: frontal, side, rear, and rollover crashes [5]. Many different models have been proposed to describe the crash process, including the finite element (FE) model and the lumped parameter model (LPM) $[6,7]$. The essential difference between these two lies in the parameters incorporated in the vehicle model: LPM uses mass, spring, and damping to describe the vehicle's structure only, while the FE model takes into consideration the geometry, material, and connection of every component of the train. 
Many researchers are currently focusing on the development of new modeling techniques that make use of Machine Learning algorithms [8-10], and, among these, we can identify research directions that fall into three main categories: the first category consists of approaches that use FE method combined with Machine Learning. Stoffel et al. [11] take into account the strain-rate and high dynamic deformation in nonlinear structural deformations and propose an intelligent finite element, where an Artificial Neural Network (ANN) is used instead of viscoelastic constitutive equations. In this way, a finite element theory is combined with Machine Learning, which substitutes a physically nonlinear constitutive law and leads to low fidelity simulation (see Figure 1(a)).

The second category includes multibody dynamics methods combined with Machine Learning algorithms. Pawlus et al. [12] propose a rebuilding oblique crash method by applying the Levenberg-Marquardt (LM) algorithm to analyze the basic concepts of rigid vehicle kinematics and dynamics in plane motion. Pawlus et al. [13] apply a nonlinear autoregressive model and a feedforward neural network to simulate the elastic-plastic characteristics of a car body. Munyazikwiye et al. [14] investigate whether it is possible to accurately estimate the basic crashworthiness parameters (e.g., acceleration severity index and maximum dynamic crushing) by using a simple piecewise LPM and a genetic algorithm. The model is firstly calibrated with impact velocity of $56 \mathrm{~km} / \mathrm{h}$, and then the crashworthiness parameters are calculated for a range of velocities $(40,48,64$, and $72 \mathrm{~km} / \mathrm{h}$ ). These methods investigate whether it is possible to accurately estimate the basic crashworthiness parameters by using the earlier proposed LPM modeling combined with Machine Learning (see Figure 1(b)).

The third category comprises data-driven direct modeling methods. Tang et al. [15] propose a data-driven train crash modeling method. They extract useful forcedisplacement curve models and predict force-displacement relations from existing $\mathrm{FE}$ simulation data through the application of a parallel stochastic forest algorithm under various crash conditions. Acar [16] proposes outlier analysis to improve the crash response accuracy of integrated prediction models based on cross validation error. Wei et al. [17] propose a robust parameter identification method using the integrated empirical mode decomposition method. They introduce a new piecewise model structure to describe the acceleration of vehicles during frontal crash, in this way allowing for an effective estimation of the crash process under different working conditions. Zhao et al. [18] propose a new adaptive neurofuzzy inference system (ANFIS) to reconstruct the process of vehicle crash and reproduce the vehicle kinematics (acceleration, velocity, and displacement) in oblique crash mode. Munyazikwiye et al. [19] develop a fuzzy logic model to predict the crash dynamics of a vehicle from an acceleration signal and analyze the level of contribution of jerk and kinetic energy to the derivation of the impact strength. Karimi et al. [20] propose a new method to reproduce the acceleration pulse during vehicle crash based on wavelet. The originality of all these methods is directly related to the exploration of the field of vehicle dynamics modeling by using Machine Learning (see Figure 1(c)).

The rapid development of modeling methods that use Machine Learning algorithms has brought advantages in terms of model complexity being greatly reduced and computational efficiency being considerably improved. However, the resulting model is still required to explore the dynamic characteristics of vehicle structure interactions in a train crash process and also to give a relevant physical explanation for data-driven methods. Therefore, the scope of this work is to build a surrogate model to explore the nonlinear spring-mass-damper of the crash process based on both experimental results and FE modeling results. Moreover, this work will have very important theoretical and application value for improving the crashworthiness and safety of train and vehicle structures.

In this paper, the CLGAN model and the nonlinear lumped parameter model are built to reproduce the train crash process (see Figure $1(\mathrm{~d})$ ). In the first step, the nonlinear lumped parameter model consisting of five key components and ten parameters is built. Then, the nonlinear time-varying parameters of the five key components in twenty different cases are estimated and predicted during the train frontal crash process by applying the CLGAN model, which shows great potential in nonlinear estimation. Finally, the train frontal crash process is estimated under different conditions, and lastly the model is verified.

\section{The Nonlinear Lumped Model}

Train crash is a complex problem in terms of the design of material, contact, and geometric nonlinearity [21, 22]. In this work, we conducted cash experiments on the energy absorbing device of the front locomotive (see Figures 2(a) and $2(\mathrm{~b})$ ). The front locomotive was equipped with anticlimbing devices and energy absorbing components. The transient impact force test system is used to measure the impact force in real time and the high-speed camera system is used to record the entire impact process. Then the sequences image motion analysis of the energy absorption device is performed. Finally, the dynamic responses such as the deformation mode, impact stroke, displacement, velocity, and acceleration during the impact process are obtained.

At the same time, the FE model of energy absorbing device (see Figures 2(c) and 2(d)) and the representative FE model (see Figures 2(e) and 2(f)) are built through the use of a four-node shell mesh to disperse the vehicle on the LSDYNA software platform. The bilinear isotropic and kinematic hardening material models are used to express the elastic and plastic properties; the translational freedom of one-end two-spring seat in the $z$-axis direction is constrained; the relative relationship between the bogie and the vehicle body is simulated. The overall Euler buckling deformation occurs at the front end of the car body; thus the rigid wall is constrained with 6 degrees of freedom to simulate it. As a result, the FE model with nonlinear large deformation is built, and subsequently we are able to obtain the FE modeling results under different velocities. 


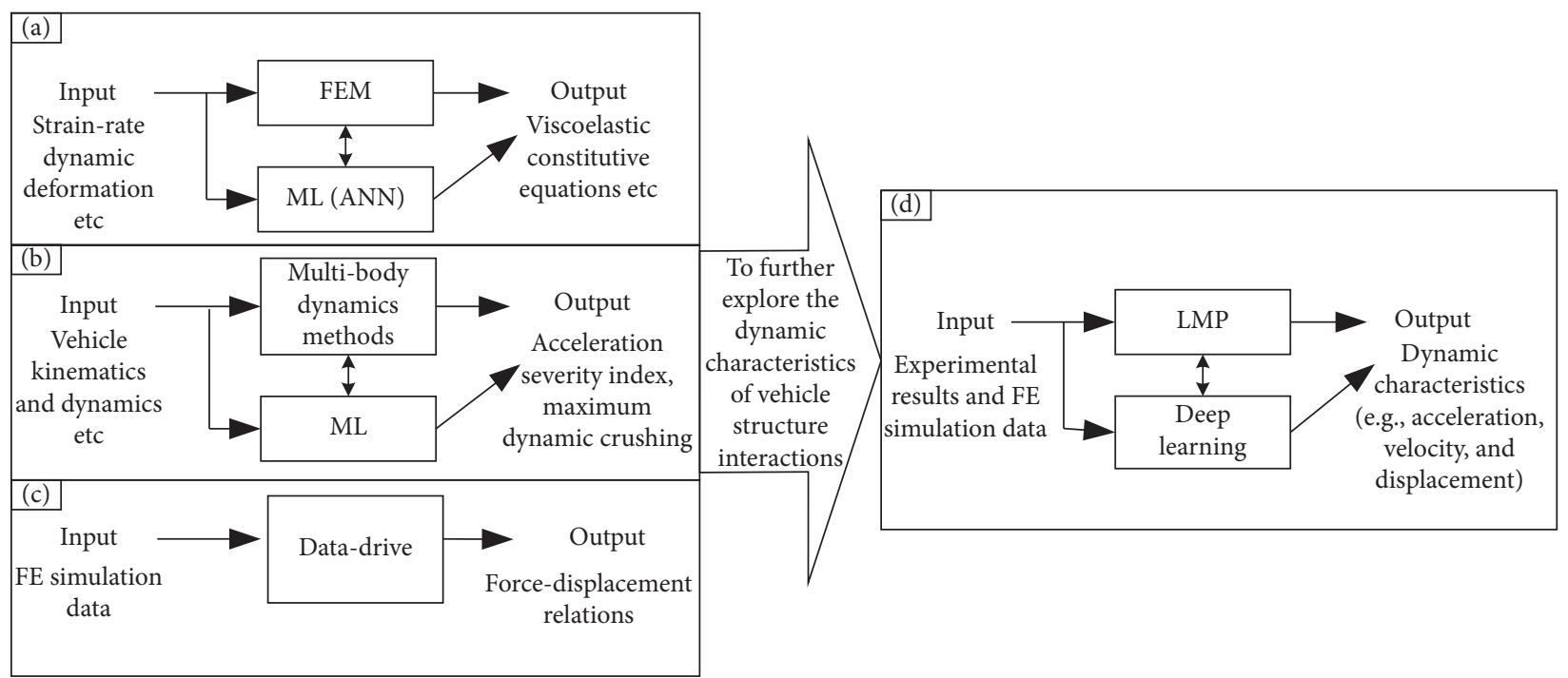

Figure 1: Mechanistic and statistical methods for mechanics problems.

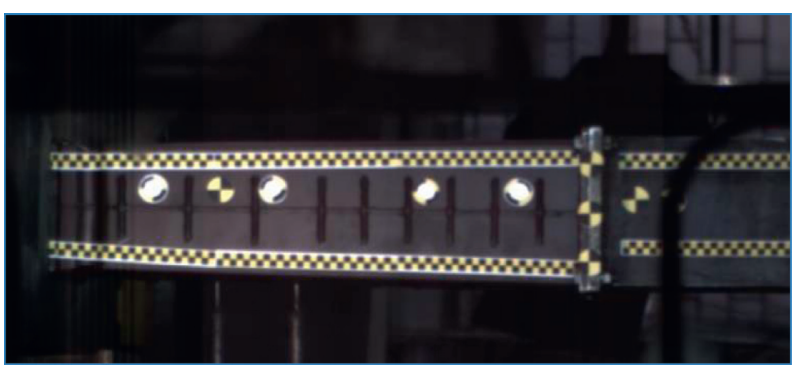

(a)

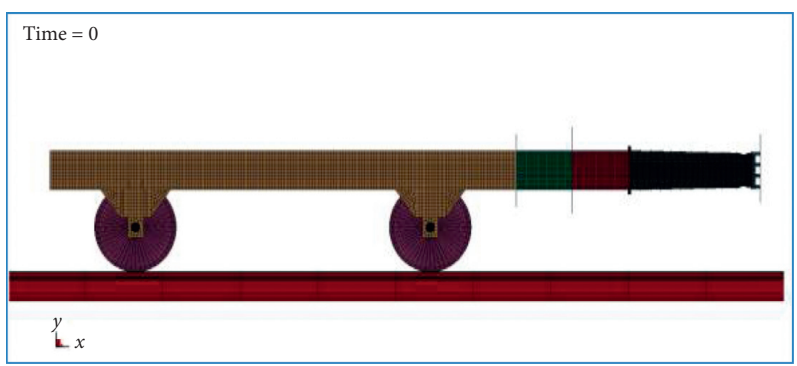

(c)

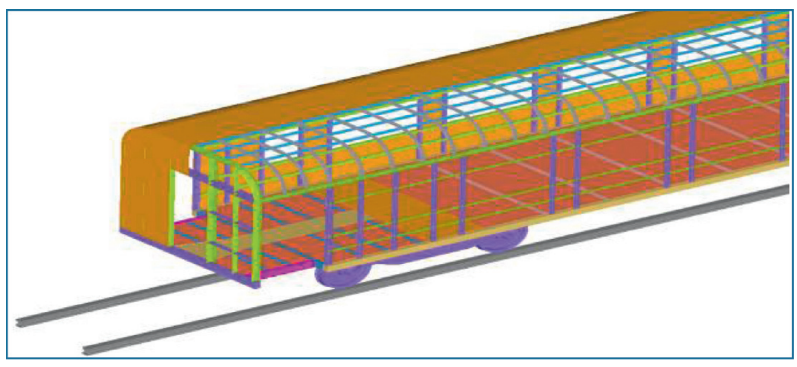

(e)

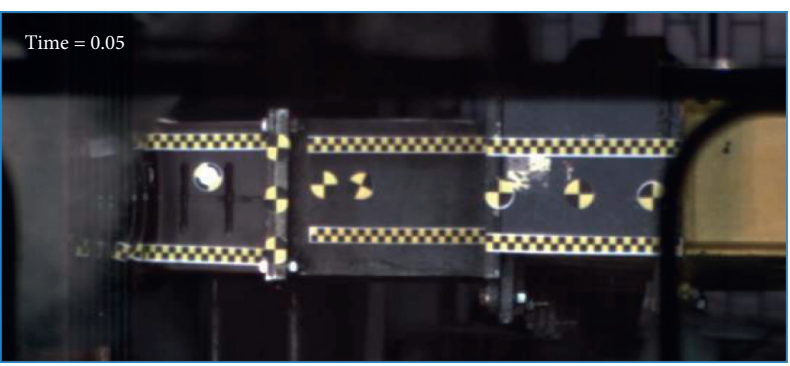

(b)

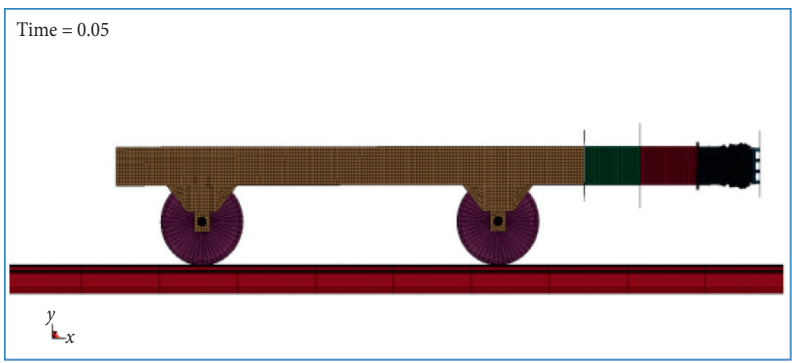

(d)

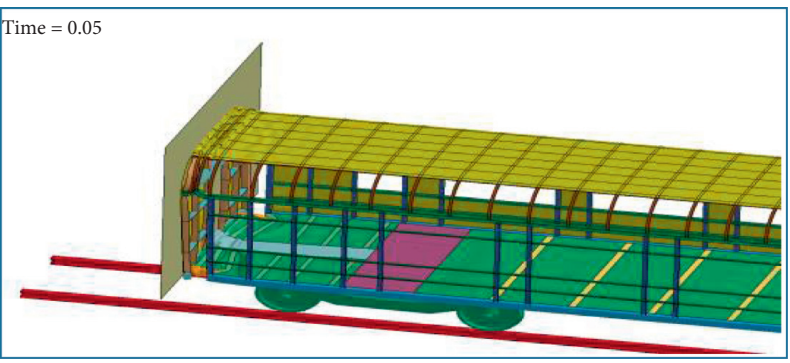

(f)

FIGURE 2: Cash experiments and FEM simulation. (a) Experimental state of energy absorbing device at initial time. (b) Experimental results of energy absorption device at initial speed of $60 \mathrm{~km} / \mathrm{h}, t=50 \mathrm{~ms}$. (c) FE model of energy absorbing device. (d) FE model simulation results of energy absorption device at initial speed of $60 \mathrm{~km} / \mathrm{h}, t=50 \mathrm{~ms}$. (e) FE model of intermediate car. (f) FE simulation results of the intermediate vehicle model at $60 \mathrm{~km} / \mathrm{h}$ and $t=50 \mathrm{~ms}$. 
In order to further accurately evaluate the nonlinear variation of the train frontal crash process, a physical model of nonlinear spring-mass-damper, which includes mass, stiffness, and damping coefficients, is built through the parameterization of this complex process (see Figure 3). Being the most common vibration system, the spring-massdamper can quantitatively describe the dynamic characteristics of the system and reveal the mathematical expressions between the system's structural and dynamic parameters. The resulting model is suitable for component modeling (creep and relaxation) as well as for vehicle frontal impact modeling.

2.1. Head Car Modeling. The nonlinear head car lumped model consists of the head car mass block and the anticlimbing energy absorber mass block, which are able to estimate and analyze the specific behavioral performance of the head car and the anticlimbing energy absorber, respectively. The nonlinear lumped model of the head car is shown in Figure 3(a). In the diagram, $M_{1}$ is the mass of the anticlimbing energy absorber, $K_{1}$ is the initial stiffness of the anticlimbing energy absorber, $C_{1}$ is the initial damping of the anticlimbing energy absorber, $M_{2}$ is the mass of the head car, $K_{2}$ is the initial stiffness of the head car, and $C_{2}$ is the initial damping of the head car. The corresponding kinematics equation is established as follows [23]:

$$
\begin{aligned}
M_{1} \ddot{x}_{1}+K_{1} x_{1}+C_{1} \dot{x}_{1}+K_{2}\left(x_{1}-x_{2}\right)+C_{2}\left(\dot{x}_{1}-\dot{x}_{2}\right) & =0, \\
M_{2} \ddot{x}_{2}-K_{2}\left(x_{1}-x_{2}\right)-C_{2}\left(\dot{x}_{1}-\dot{x}_{2}\right) & =0 .
\end{aligned}
$$

We sum up equation (1) and rearrange to get

$$
M \ddot{X}+C \dot{X}+K X=0 \text {. }
$$

The mass, the stiffness, and the damping coefficients of the nonlinear lumped model are found to be $M=\left[\begin{array}{l}M_{1} \\ M_{2}\end{array}\right]$, $X=\left[\begin{array}{l}x_{1} \\ x_{2}\end{array}\right], K=\left[\begin{array}{cc}K_{1}+K_{2}-K_{2} \\ -K_{2} & K_{2}\end{array}\right]$, and $C=\left[\begin{array}{cc}C_{1}+C_{2} & -C_{2} \\ -C_{2} & C_{2}\end{array}\right]$.

In this work, the displacement, velocity, and acceleration, which are obtained from finite element method at different initial velocity, are substituted into equation (2), followed by computation of spring stiffness and damping coefficients at each timestep. On the basis of this data analysis, we are able to deduce the performance of the stiffness and the damping coefficients in the given cases.

2.2. Intermediate Vehicle Modeling. The estimation and analysis of the specific performance of the coupler buffer system in trains require building the nonlinear intermediate vehicle lumped model, which consists of two vehicle mass blocks as shown in Figure 3(b). In the diagram, $M_{i}$ and $M_{i+1}(i=2,3,4)$ are the masses of two intermediate vehicles, whereas $K$ and $C$ are the initial stiffness and initial damping coefficient of the coupler buffer device, respectively. The following equations of motion (EOM) are established:

$$
\begin{aligned}
M_{i} \ddot{x}_{i}+K_{i}\left(x_{i}-x_{i+1}\right)+C_{i}\left(\dot{x}_{i}-\dot{x}_{i+1}\right) & =0, \\
M_{i+1} \ddot{x}_{i+1}-K_{i}\left(x_{i}-x_{i+1}\right)-C_{i}\left(\dot{x}_{i}-\dot{x}_{i+1}\right) & =0 .
\end{aligned}
$$

We sum up equation (3) and rearrange to get

$$
\begin{aligned}
& q=\left(x_{i}-x_{i+1}\right), \\
& \dot{q}=\left(\dot{x}_{i}-\dot{x}_{i+1}\right) .
\end{aligned}
$$

By substituting equation (4) into equation (3), we obtain the following EOM:

$$
\ddot{q}+C_{i}\left(\frac{1}{M_{i}}+\frac{1}{M_{i+1}}\right) \dot{q}+K_{i}\left(\frac{1}{M_{i}}+\frac{1}{M_{i+1}}\right) q=0 .
$$

We sum up equation (5) and rearrange to get

$$
\begin{gathered}
C=C_{i}\left(\frac{1}{M_{i}}+\frac{1}{M_{i+1}}\right), \\
K=K_{i}\left(\frac{1}{M_{i}}+\frac{1}{M_{i+1}}\right) .
\end{gathered}
$$

Finally, the characteristic equation of the nonlinear lumped model is defined as

$$
\ddot{q}+C \dot{q}+K q=0 .
$$

2.3. Train Modeling. The nonlinear train lumped model with multiple vehicle mass blocks is built to estimate and analyze the specific behavioral performance of the locomotive head and coupler buffer devices, respectively. Figure 3(c) illustrates the nonlinear lumped model of a train with four vehicles. In the diagram, $M_{1}$ is the mass of anticlimbing energy absorber, $M_{2}$ is the mass of the head car, and $M_{3}, M_{4}$, and $M_{5}$ are the masses of three intermediate vehicles; $K_{1}$ is the initial stiffness of anticlimbing energy absorber, $K_{2}$ is the initial stiffness of the head car, and $K_{3}, K_{4}$, and $K_{5}$ are the initial stiffness coefficients of the intermediate vehicle; $C_{1}$ is the initial damping coefficient of anticlimbing energy absorber, $C_{2}$ is the initial damping coefficient of the head car, and $C_{3}, C_{4}$, and $C_{5}$ are the initial stiffnesses of the intermediate vehicle, and the ten parameters have nonlinear characteristics. The differential equations of train motion with masses $M_{1}, M_{2} \ldots M_{5}$ can be expressed by using D'Alembert's principle [24]: 


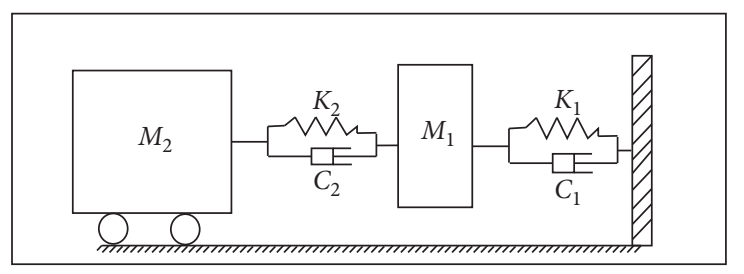

(a)

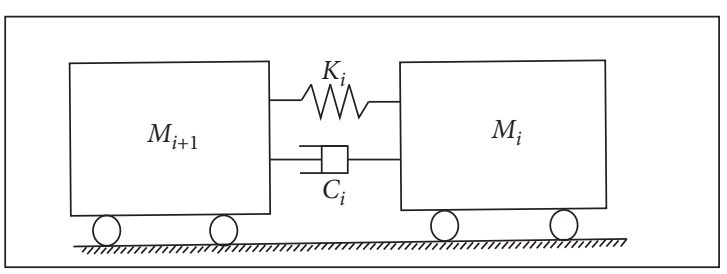

(b)

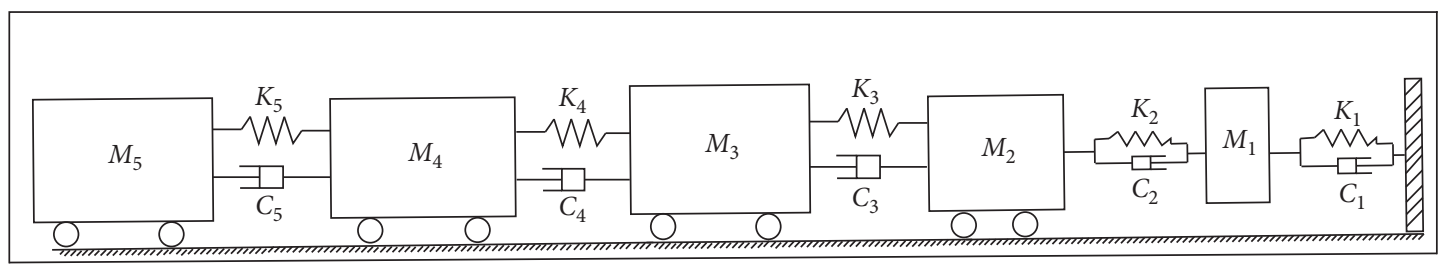

(c)

FIGURE 3: Nonlinear lumped model of (a) the head car, (b) intermediate vehicle, and (c) train.

$$
\begin{array}{r}
M_{1} \ddot{x}_{1}+C_{1} \dot{x}_{1}+C_{2}\left(\dot{x}_{1}-\dot{x}_{2}\right)+K_{1} x_{1}+K_{2}\left(x_{1}-x_{2}\right)=0, \\
M_{2} \ddot{x}_{2}+C_{2}\left(\dot{x}_{2}-\dot{x}_{1}\right)+C_{3}\left(\dot{x}_{2}-\dot{x}_{3}\right)+K_{2}\left(x_{2}-x_{1}\right)+K_{3}\left(x_{2}-x_{3}\right)=0, \\
M_{3} \ddot{x}_{3}+C_{3}\left(\dot{x}_{3}-\dot{x}_{2}\right)+C_{4}\left(\dot{x}_{3}-\dot{x}_{4}\right)+K_{3}\left(x_{3}-x_{2}\right)+K_{4}\left(x_{3}-x_{4}\right)=0, \\
M_{4} \ddot{x}_{4}+C_{4}\left(\dot{x}_{4}-\dot{x}_{3}\right)+C_{5}\left(\dot{x}_{4}-\dot{x}_{5}\right)+K_{4}\left(x_{4}-x_{3}\right)+K_{5}\left(x_{4}-x_{5}\right)=0, \\
M_{5} \ddot{x}_{5}+C_{5}\left(\dot{x}_{5}-\dot{x}_{4}\right)+K_{5}\left(x_{5}-x_{4}\right)=0 .
\end{array}
$$

The nonlinear variation of stiffness and damping coefficients that changes with time can be calculated by substituting the dynamic characteristics of two adjacent moments into D'Alembert's principle, and this lays the foundation for further exploration of the nonlinear lumped model.

Generally, the maximum train crash speed is $100 \mathrm{~km} / \mathrm{h}$; thus, in this paper, we choose the initial velocity to be in the range between $5 \mathrm{~km} / \mathrm{h}$ and $100 \mathrm{~km} / \mathrm{h}$. In order to further explore the dynamic characteristics of trains in different initial cases, we choose a step of $5 \mathrm{~km} / \mathrm{h}$ to perform calculations, so that the dynamic performance of 20 different initial states is analyzed with full consideration of calculation and sufficiency, meaning that there is enough data to learn, and the amount of calculation is relatively low. Therefore, we are able to obtain variations for 10 nonlinear lumped model parameters under different initial conditions. Finally, a perusal of the literature $[25,26]$ revealed the specific parameters of the initial train model to be set, as can be seen in Table 1 .

The flowchart of the nonlinear lumped modeling is shown in Figure 4. In the first stage, a reference anticlimbing energy absorber is taken for crash tests, and the results of the experiments are used to validate the FE analysis. Then, the validated FE model conducts numerical tests on 20 different initial velocities using the LS-DYNA solver, and train crash characteristics and corresponding nonlinear parameters are stored in a knowledge database. In the second stage, the nonlinear relationship between train crash nonlinear parameters is calculated by using dynamic characteristics in twenty initial conditions (input), whereas the nonlinear parameters under different initial conditions (output) are established through the CLGAN model. In the third stage, the estimated nonlinear parameters are substituted into the nonlinear lumped model, allowing the quick and effective computation of train crash dynamic characteristics under any condition.

The neural network model, which we called CLGAN model in this paper, is validated through comparison by analyzing the original data obtained from the FE model based on the LS-DYNA platform. In this work, a 10-parameter nonlinear lumped model of the train is built based on D'Alembert's principle; then the crash process is reconstructed by evaluating and predicting the variation of nonlinear parameters properties. The specific analysis of the spring stiffness and damping coefficients under different conditions will be introduced in Section 4 .

\section{CLGAN Model}

3.1. Data Preprocessing. Abundant performance data of nonlinear spring stiffness and damping coefficients under different conditions could be obtained by combining experimental analysis with FE analysis. However, there are many uncertainties in the actual calculation, which leads to the randomness of the generated data. To accurately evaluate the characteristics of the nonlinear parameters, it is necessary to recognize and process the abnormal data. In this work, we mainly apply the three-sigma rule for anomaly detection and processing of raw data and use Fourier transform for filtering in the frequency domain in order to correct it.

The three-sigma rule [27], also known as PauTa Criterion, determines an interval according to the calculated probability value. Test data are considered to be a random error when it lies within this interval and as a gross error 
TABle 1: Description of the train parameters.

\begin{tabular}{lccc}
\hline Parameter & Description & Initial design & \\
\hline$M_{2}$ & Head car masses & 37000 & Unit \\
$M_{3}, M_{4}, M_{5}$ & Intermediate vehicle masses & 34000 & $\mathrm{~kg}$ \\
$M_{1}$ & Anticlimbing device masses & 75 & $\mathrm{~kg}$ \\
$K_{3}, K_{4}, K_{5}$ & Initial stiffness of coupler buffer device & $0.8 * 10^{\wedge} 6$ & $\mathrm{~kg}$ \\
$C_{3}, C_{4}, C_{5}$ & Initial damping coefficient of coupler buffer device & $5.2 * 10^{\wedge} 4$ & $\mathrm{~N} / \mathrm{m}$ \\
$K_{2}$ & Initial stiffness of head car & $3.1 * 10^{\wedge} 7$ & $\mathrm{Ns} / \mathrm{m}$ \\
$K_{1}$ & Initial stiffness of anticlimbing device & $1.0 * 10^{\wedge} 5$ & $\mathrm{~N} / \mathrm{m}$ \\
$C_{2}$ & Initial damping coefficient of head car & $6.0 * 10^{\wedge} 5$ & $\mathrm{~N} / \mathrm{m}$ \\
$C_{1}$ & Initial damping coefficient of anticlimbing device & $2.0 * 10^{\wedge} 4$ & $\mathrm{Ns} / \mathrm{m}$ \\
$V_{0}$ & Initial velocity of tests & $5 / 10 / 15 / 20 / 25 / 30 / 35 / 40 / 45 / 50 / 55 / 60 / 65 / 70 / 75 / 80 / 85 / 90 / 95 /$ & $\mathrm{Ns} / \mathrm{m}$ \\
\end{tabular}
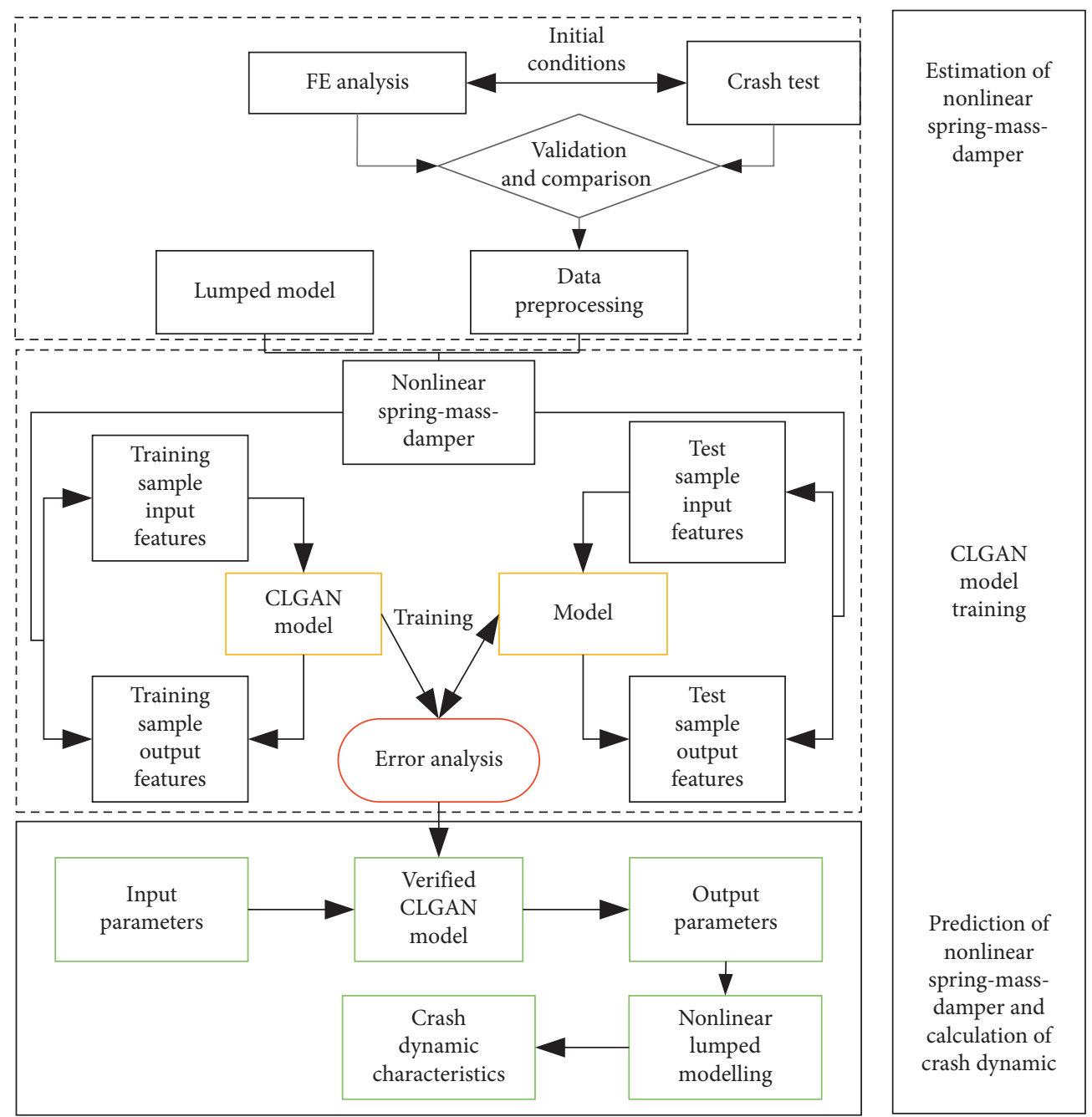

Figure 4: Modeling flowchart of the nonlinear lumped model.

when it falls beyond the specified interval. In this way, we can distinguish and judge abnormal values. The main calculation steps are as follows:

(1) Calculate the standard deviation of the original data.

(2) Compare the absolute difference between each sampling point and the mean value with three times the standard deviation. The data point is eliminated if it is greater than three times the standard deviation. The calculation formula is as follows:

$$
\left|x_{i}-\bar{x}\right|>3 \sqrt{\frac{\left(x_{1}-\bar{x}\right)^{2}+\left(x_{2}-\bar{x}\right)^{2}+\cdots+\left(x_{n}-\bar{x}\right)^{2}}{n-1}} .
$$


(3) Repeat step 1 until all outliers are identified and processed.

The data we have is time-domain information, and, in fact, some information is hidden in the frequency-domain part. Therefore, in this work, we apply a mathematical transformation method, such as Fourier transform [28], Wavelet transform [29], and Hilbert transform [30], into the time frequency analysis of train crash signals to obtain the most apparent information. In this work, Fourier transform [28], a kind of reversible transformation, is mainly used to obtain the frequency-domain information from the spring stiffness and damping coefficients data obtained previously, so as to realize the frequency-domain filtering. The Fourier transform formula is

$$
X(f)=\int_{-\infty}^{\infty} x(t) \cdot e^{-2 j \pi f t} \mathrm{~d} t .
$$

Fourier inverse transform is applied to the nonlinear spring stiffness and damping coefficients obtained from the CLGAN model, and the final time-domain information is obtained. The formula is as follows:

$$
x(t)=\int_{-\infty}^{\infty} X(f) \cdot e^{2 j \pi f t} \mathrm{~d} t .
$$

In the formula, $t$ represents time, $f$ denotes frequency, $x$ corresponds to signal data in the time domain, and $X$ represents signal data in the frequency domain.

3.2. Model Architecture. Due to the strong nonlinearity of the parameters in complicated train crash processes, it is difficult to balance the complexity of models and the accuracy of estimation. A CLGAN model, combining Generative Adversarial Network (GAN), Convolutional Neural Network (CNN), and Long-Short-Term-Memory (LSTM) neural network, is built to explore the variation rule of nonlinear spring-mass-damper during the crash process based on both experimental results and FE modeling results.

In this work, nonlinear parameters can be estimated through the analysis of the train crash dynamic characteristics data (e.g., displacement, velocity, and acceleration) obtained from general FE modeling. We take initial conditions (e.g., velocity and time) as input data of CLGAN and the variation of nonlinear parameters over time as the output of CLGAN. We use LSTM as the generator $G$ to generate the adversarial neural network and use CNN as the discrimination $\mathrm{D}$ to make same distribution of the generated value coincide with the nonlinear parameters. Then the corresponding relationship between the initial conditions and the train crash nonlinear parameters can be determined.

The nonlinear spring stiffness and damping coefficients are a series of data over time, so we prefer $1 \mathrm{DCNN}$, which mainly includes a $1 \mathrm{D}$ convolution layer (Cov1D layer), a pooling layer, a fully connected layer, and a nonlinear activation function [31]. In this network, $X=$ $\left(x_{1}, x_{2}, \ldots, x_{n}, c l\right)$ is input vector, where $x_{n} \in R^{d}$ and $c l \in R$ represent the feature and the label of the class, respectively. A series of new features $f$ can be obtained by convolving the input data. These features are the input of the output layer, and then the posterior probabilities of each category are obtained, defined as follows:

$$
O_{t}=\operatorname{soft} \max \left(V_{0} * \tanh \left(\omega^{f} x_{i, i+f-1}+b\right)+b_{0}\right) \text {, }
$$

where $b, b_{0} \in R$ is a bias term.

The LSTM network is a Recurrent Neural Network (RNN). This network uses a control gate mechanism and includes memory cells, input gates, output gates, and forget gates [32]. In the LSTM network, the cell candidate state value at time $t$ is the input gate value, the activation value of the forget gate at time $t$ can be calculated, and then the cell state updated value $C_{t}$ at time $t$ can be calculated.

$$
\begin{aligned}
C_{t}= & \delta\left(W_{i} *\left(X_{t}, h_{t-1}\right)+b_{i}\right) * \tanh \left(W *\left(X_{t}, h_{t-1}\right)+b_{c}\right) \\
& +\delta\left(W *\left(X_{t}, h_{t-1}\right)+b_{f}\right) * C_{t-1} .
\end{aligned}
$$

Then, the value of the output gate is obtained, and the calculation formula as follows:

$$
O_{t}=\delta\left(W_{0} *\left(X_{t}, h_{t-1}\right)+b_{o}\right) * \tanh \left(C_{t}\right) .
$$

GAN is a minimal maximum confrontation game between discriminator $D$ and generator $G$, which is kind of minimal maximization problem [33]. To optimize the important parameters and improve the ability of generator $G$, the distribution of generated samples should gradually approach the real data samples, so the specific objective function can be described as

$$
\begin{aligned}
\min _{G} \max _{D} V(D, G)= & E_{X \sim \text { Pdate }}(x)[\log D(x)] \\
& +E_{z \sim p z(z)}[\log (1-D(G(z)))],
\end{aligned}
$$

where $E_{X \sim P \text { date }}$ and $E_{X \sim z}$ represent the expected loss of real data and the distribution of noise $z$, respectively.

Finally, the important parameters and performance of the generator can be optimized and improved through alternating iteration. Then the corresponding relationship between the initial conditions and the train crash nonlinear parameters can be determined. So the nonlinear spring stiffness and damping coefficients can be predicated under other conditions, and the dynamic characteristics can be derived through using Newmark- $\beta$ method. Therefore, the train crash dynamic characteristics are accurately estimated during the train crash processes, and the crash processes under different given conditions can be described effectively.

In the CLGAN model, we set the discriminator initial learning rate to 0.008 and the generator learning rate to 0.004 to ensure the stability of the system. For training more deeper and complex networks, we generally use an optimizer with adaptive learning rate to converge faster, so we chose Adam as the generator optimizer, which is suitable for most nonconvex optimizations, large datasets, and high-dimensional spaces; and we choose RMSprop as the discriminator optimizer, which is suitable for handling nonstationary targets and works well for RNN; the ReLU activation function is replaced by a LeakyReLU layer. See the CLGAN algorithm pseudocode for a detailed overview of the process in Algorithm 1 [34]. 
For number of training iterations do:

For $K$ steps do:

Sample mini batch of $m$ noise samples $\left\{z^{(1)}, \ldots, z^{(m)}\right\}$ from noise prior $P_{g}(z)$

Sample mini batch of $m$ examples $\left\{x^{(1)}, \ldots, x^{(m)}\right\}$ from data applying LSTM net to generate distribution $P_{\text {data }}(x)$

Sample mini batch of $m$ examples $\left\{y^{(1)}, \ldots, y^{(m)}\right\}$ from data applying CNN as discriminator

The cost function of the calculation discriminator:

$J_{d}=(1 / m) \sum_{i=1}^{m}\left[\log D\left(x^{(i)}\right)+\log \left(1-D\left(G\left(z^{(i)}\right)\right)\right)\right]$

Update the parameters of the discriminator through RMSprop gradient drop algorithm:

End for $\theta_{d}=\operatorname{Adam}\left(\nabla \theta_{d}\left(J_{d}\right), \theta_{d}\right)$

Sample mini batch of $m$ noise samples $\left\{z^{(1)}, \ldots, z^{(m)}\right\}$ from noise prior

The cost function of the calculation generator: $J_{g}=(1 / m) \sum_{i=1}^{m} \log \left(1-D\left(G\left(z^{(i)}\right)\right)\right)$

Update the parameters of the generator through Adam gradient drop algorithm: End for $\theta_{g}=\operatorname{Adam}\left(\nabla \theta_{g}\left(J_{g}\right), \theta_{g}\right)$

AlGORITHM 1: CLGAN algorithm: mini-batch stochastic gradient descent training of generative adversarial net.

The training process of CLGAN neural network is shown in Figure 5. There are two kinds of training samples, the real pair and the fake pair. To adapt the dataset files to our waveform generation purposes, we downsample the original data to evaluate the nonlinear spring stiffness and damping coefficients. Furthermore, twenty different initial velocities $(5 \mathrm{~km} / \mathrm{h}, 10 \mathrm{~km} / \mathrm{h}, 15 \mathrm{~km} / \mathrm{h}, 20 \mathrm{~km} / \mathrm{h}, 25 \mathrm{~km} / \mathrm{h}, 30 \mathrm{~km} / \mathrm{h}$, $35 \mathrm{~km} / \mathrm{h}, 40 \mathrm{~km} / \mathrm{h}, 45 \mathrm{~km} / \mathrm{h}, 50 \mathrm{~km} / \mathrm{h}, 55 \mathrm{~km} / \mathrm{h}, 60 \mathrm{~km} / \mathrm{h}$, $65 \mathrm{~km} / \mathrm{h}, 70 \mathrm{~km} / \mathrm{h}, 75 \mathrm{~km} / \mathrm{h}, 80 \mathrm{~km} / \mathrm{h}, 85 \mathrm{~km} / \mathrm{h}, 90 \mathrm{~km} / \mathrm{h}$, $95 \mathrm{~km} / \mathrm{h}$, and $100 \mathrm{~km} / \mathrm{h}$ ) are considered.

During training, we extract chunks of waveforms with a sliding window of approximately a tenth of a second of speech $(67840 \times 10$ samples) every $500 \mathrm{~ms}$ (50\% overlap). During the testing stage, we slide the window with no overlap through the whole duration of our test data and concatenate the results at the end of the stream. In both training and testing, we apply a high-frequency preemphasis filter with coefficient of 0.95 to all input samples (during testing, the output is deemphasized accordingly).

Regarding the architecture, the generator $G$ is composed of 5 LSTM layers. The number of filters per layer increases so that the depth gets larger as the width (i.e., duration of signal in time) gets narrower. Each of the five neuron layers has a size of 64, while the corresponding training parameters are set to $67840,33024,33024,33024$, and 33024, respectively. There, we sample the noise samples $z$ from our prior $1 \times 385$ dimensional normal distribution $N(0, I)$. As previously mentioned, the decoder part of $\mathrm{G}$ mirrors the encoder by having the same filter widths and the same number of filters per layer. However, due to skip connections and the addition of the latent vector, the number of feature maps in every layer is doubled. The network D is composed of 3 one-dimensional convolutional layers, and it follows the conventional topology of a convolutional classification network. The main characteristics are as follows: (1) it gets two input channels of 67840 samples; (2) it uses virtual batch norm before LeakyReLU nonlinearities; (3) in the last activation layer, there is a one-dimensional convolution layer with one filter of width 1 which does not downsample the hidden activations. This last stage (3) allows for the reduction of the number of parameters required for the final classification neuron, which is fully connected to all hidden activations in a linear way. In conclusion, this CLGAN model abandons the complex process of sampling and processing in the traditional stage and has a good regress generating function for the nonlinear prediction.

\section{Estimation and Prediction of Nonlinear Spring-Mass-Damping}

Nonlinear parameters can be estimated through the analysis of the train crash dynamic characteristics data (e.g., displacement, velocity, and acceleration) obtained from general FE modeling. In this work, these parameters are used as input data to the CLGAN model, and the variation of nonlinear spring stiffness and damping coefficients under different initial conditions over time is predicted and is the output data of the CLGAN model. To make a simple comparative analysis, twenty different initial velocities are used for this purpose, and this not only ensures sufficient sample capacity but also accurately describes the nonlinear characteristics of the spring stiffness and damping coefficients.

4.1. Estimation and Analysis of Nonlinear Spring-MassDamping. Twenty initial crash velocities are picked in the range from $5 \mathrm{~km} / \mathrm{h}$ to $100 \mathrm{~km} / \mathrm{h}$. Then the stiffness of the head car $\left(K_{1}\right)$, the anticlimbing energy absorption device $\left(K_{2}\right)$, the coupler buffer device $\left(K_{3}, K_{4}\right.$, and $\left.K_{5}\right)$, the damping coefficient $\left(C_{1}\right)$, the head car damping coefficient $\left(C_{2}\right)$, and the coupler buffer coefficients $\left(C_{3}, C_{4}\right.$, and $\left.C_{5}\right)$ can be calculated accordingly. The parameters' variation curves are obtained, and a comparative analysis is carried out to explore the patterns.

In this work, the physical parameters of the head car, anticlimbing device, and coupler buffer devices at different initial speeds are analyzed. The results show that the head car 


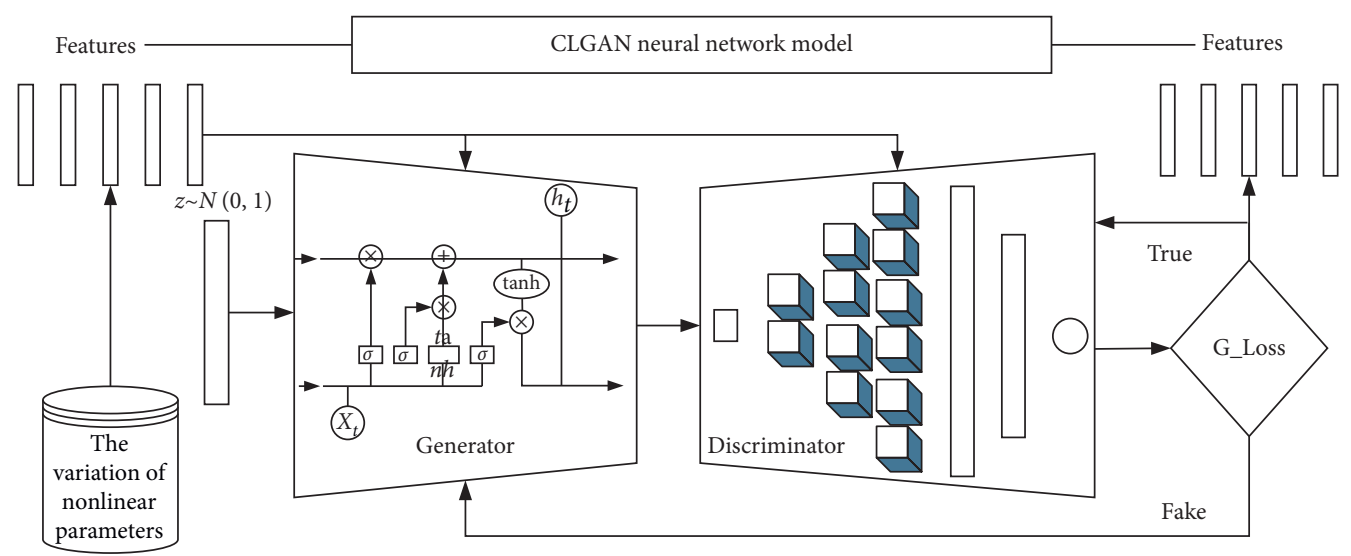

Figure 5: CLGAN model.

resists deformation at the moment of mid-high-speed crash; after that, the anticlimbing device and coupler buffer devices resist deformation. Therefore, we can conclude that the anticlimbing energy absorber performance is best at middle and low speed, whereas a delayed state can be found at high speed by comparing and analyzing the parameters variation. In addition, this method can provide new guidance to further improve the performance of anticlimbing and coupler buffer devices.

After evaluating the results obtained at 20 initial velocities, the derived nonlinear spring stiffness and damping coefficients are plugged in as input to the CLGAN model to obtain more precise parameters variation over time. The output values through training correspond to the nonlinear spring stiffness and damping calculated at the given speeds. To illustrate the results effectively, the initial velocities of $10 \mathrm{~km} / \mathrm{h}, 35 \mathrm{~km} / \mathrm{h}, 60 \mathrm{~km} / \mathrm{h}$, and $85 \mathrm{~km} / \mathrm{h}$ are chosen, and the comparison results obtained by estimation and prediction contrastive analysis are shown in Figures 6-10.

As can be seen in Figure 6, when the anticlimbing device collides at a low initial speed, the stiffness and damping values reach their maximum at the same moment at $0.06 \mathrm{~s}$ : at this time, the stiffness value is much larger than the damping one, indicating that the anticlimbing device starts to resist the main deformation [35]. As the speed increases, stiffness and damping values quickly reach their maximum. When the crash occurs at a higher initial speed, the stiffness and damping values reach their maximum at about $0.05 \mathrm{~s}$ : the damping value at this time is far greater than the stiffness one, meaning that the anticlimbing device absorbs the main energy. In Figure 7, it can be clearly seen that when the head car collides at a high speed, the leading vehicle's stiffness and damping values change sharply, indicating that serious damage occurred at this time [36]. Figure 8 shows that when the initial crash speed is low, the stiffness and damping values have multiple high peaks, indicating that the coupler buffer device 2 has a continuous buffering effect at this time, and the energy absorption effect is the best at this speed. As the speed increases, the buffering effect of the coupler buffer device 2 decreases. As can be seen in Figure 9, when the initial crash speed is low, the stiffness value continuously fluctuates, indicating that coupler buffer device 3 plays a continuous buffering role at this time. When the initial crash speed is medium at $35 \mathrm{~km} / \mathrm{h}$, the damping coefficient of coupler buffer device 3 has reached its maximum value, and this indicates that the energy absorption effect of the coupler buffer device is the best at this speed [37]. However, the buffer effect of coupler buffer device 3 will decrease as the speed increases. Finally, from Figure 10, we can see that when the initial crash speed changes from low to high speed, the stiffness and damping values of coupler buffer device 4 always fluctuate. In addition, through a comparative analysis of the stiffness and damping parameters of the anticlimbing device, it can be seen that the predicted values completely match with the trends of the evaluation values. Furthermore, the results well verify that the CLGAN model not only has sufficient model capacity and good training accuracy but also has high generalization accuracy.

4.2. Estimation of CLGAN Model. To verify the accuracy of the prediction results, the goodness of fit, called $R$-squared (the closer to 1 the value of $R^{2}$ is, the better the fitting is), of $K_{1}, C_{1}, K_{2}, C_{2}, K_{3}, C_{3}, K_{4}, C_{4}, K_{5}$, and $C_{5}$ is $0.993,0.988,0.822$, $0.866,0.761,0.77,0.914,0.795,0.819$, and 0.821 , respectively, as can be seen in Figure 11(a). Also, the CLGAN model and RNN model are both trained in order to compare these two methods with the original data, as shown in Figure 11(b). From Figure 11(a), it can be observed that the goodness of fit of the 10 different spring-mass-damper models obtained by applying the CLGAN model is very well; at the same time, the variation of 10 nonlinear spring-mass-damper models is accurately evaluated. From Figure 11(b), it can be seen that both CLGAN and RNN are well trained by comparing the two methods, and CLGAN can better describe the variation of nonlinear spring damping compared with RNN model. Furthermore, the CLGAN method is more accurate and valid than the traditional method.

This iteration process is run sequentially for all the training datasets sample by sample, which is defined as one "epoch" iteration. The algorithm proceeds in this fashion until the error decreases below some acceptable threshold or until the error stops decreasing over possibly hundreds of epochs. The discriminator loss curves and the adversarial 

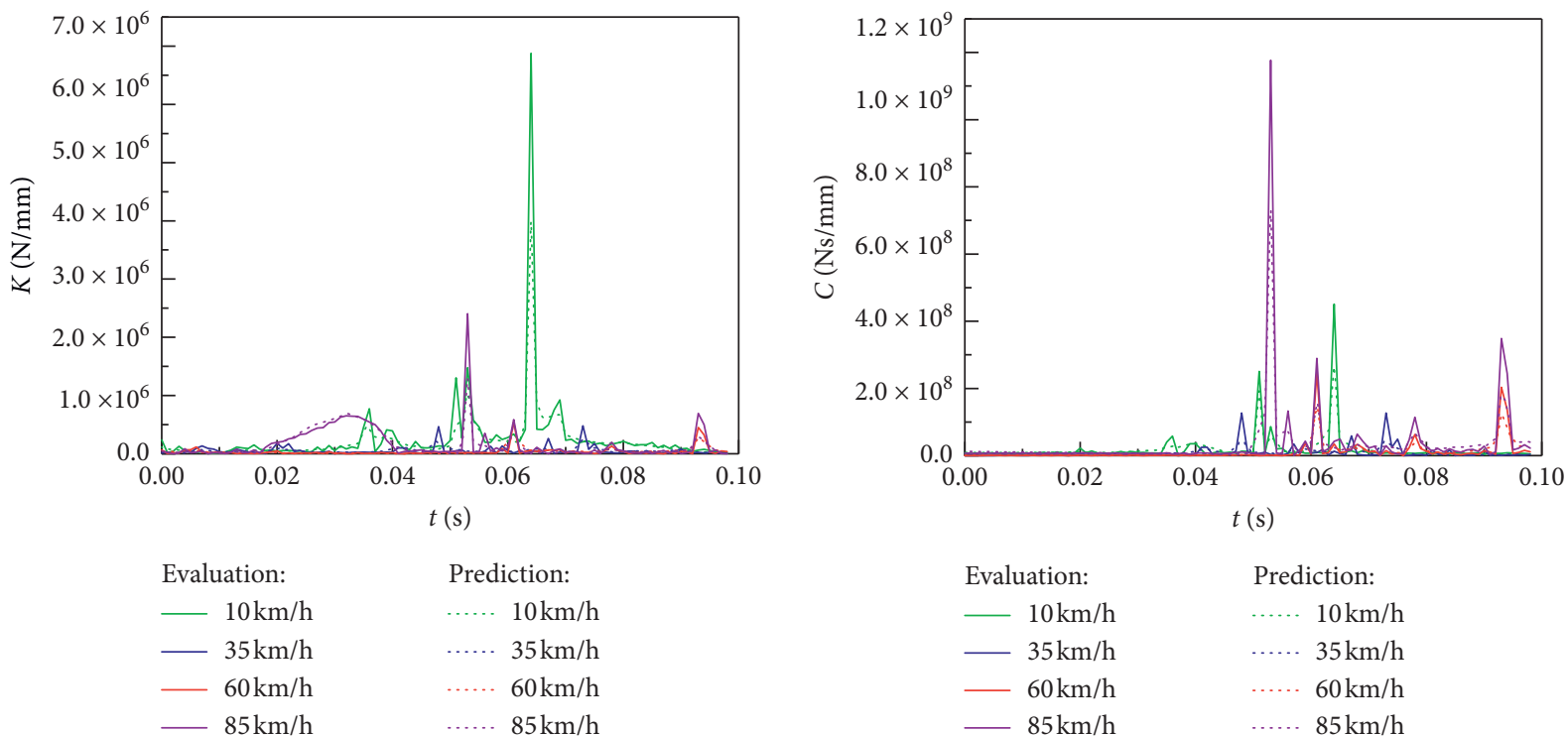

FIgURE 6: Anticlimbing device comparisons of estimation and prediction.
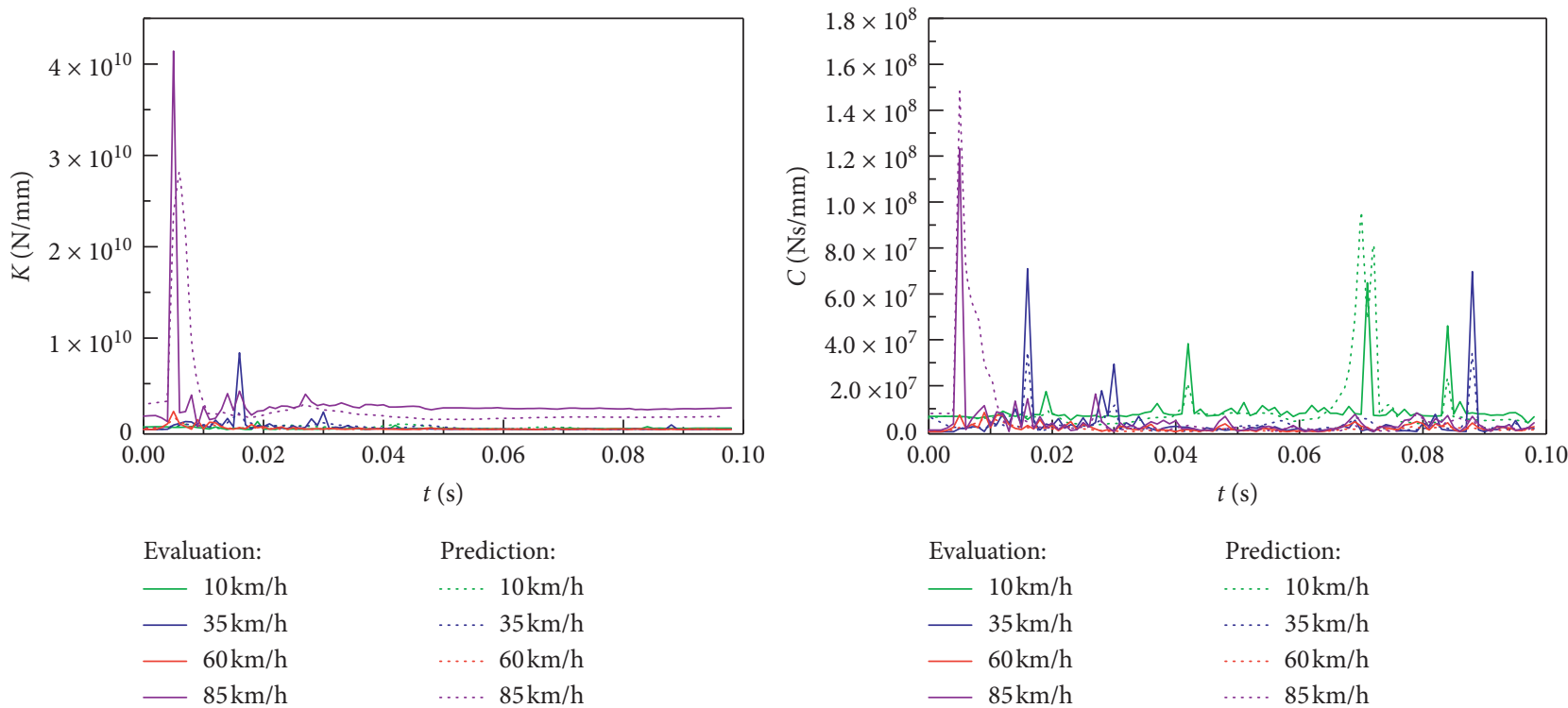

Prediction:

..... $10 \mathrm{~km} / \mathrm{h}$

Evaluation:

Prediction:

$10 \mathrm{~km} / \mathrm{h}$

.... $10 \mathrm{~km} / \mathrm{h}$

$-35 \mathrm{~km} / \mathrm{h}$

..... $35 \mathrm{~km} / \mathrm{h}$

$60 \mathrm{~km} / \mathrm{h}$ $60 \mathrm{~km} / \mathrm{h}$ ..... $85 \mathrm{~km} / \mathrm{h}$

$-85 \mathrm{~km} / \mathrm{h}$

… $85 \mathrm{~km} / \mathrm{h}$

Figure 7: Head car comparisons of estimation and prediction.

generator loss curves can be obtained after 1000 iterations, as shown in Figures 12(a) and 12(b).

Through a comparison of results, it can be seen that the predicted values by the Deep Learning model are consistent with the trend of the estimation results. Its goodness of fit is well, which indicates that the model has high accuracy. In conclusion, the CLGAN model is effective and is able to provide a good basis for obtaining the crash dynamic characteristics in Section 5.

\section{Modeling Validation}

Based on the information presented in Sections 2 and 4, ten parameters, including spring stiffness and damping coefficients, are predicted through the use of the CLGAN model. Then the displacement, velocity, and acceleration of five key components at the initial speed of $60 \mathrm{~km} / \mathrm{h}(16.67 \mathrm{~m} / \mathrm{s})$ are estimated and predicted by the Newmark- $\beta$ method [38] during the crash process.

5.1. Anticlimbing Drive Model. In this section, the anticlimbing drive model is analyzed: with the anticlimbing device crash dynamic characteristics are simulated using the CLGAN model and the Newmark- $\beta$ method. The results of the simulation are shown in Figure 13.

In order to verify the accuracy of the method, we compare anticlimbing device's crash dynamic characteristics, such as 

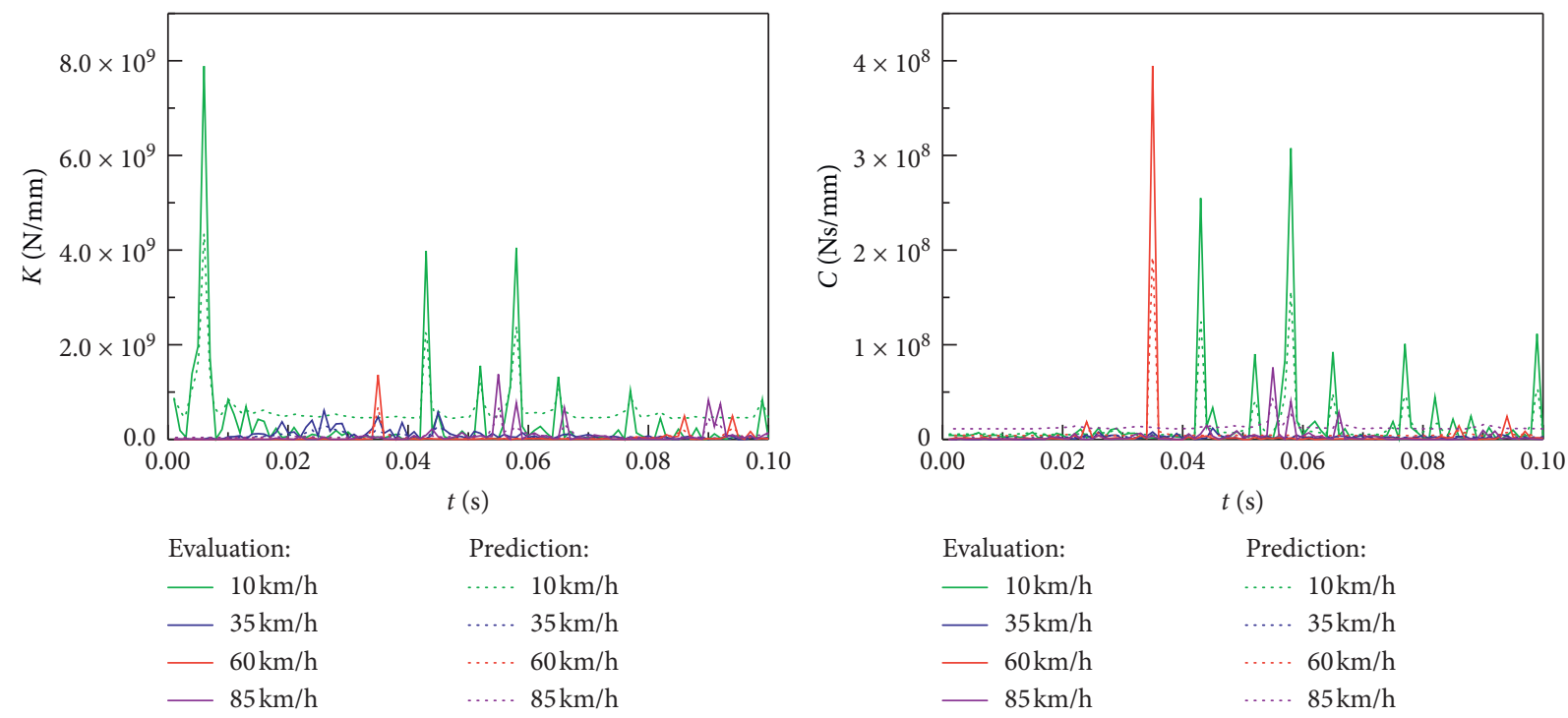

FIgURE 8: Coupler buffer device 2 comparisons of estimation and prediction.
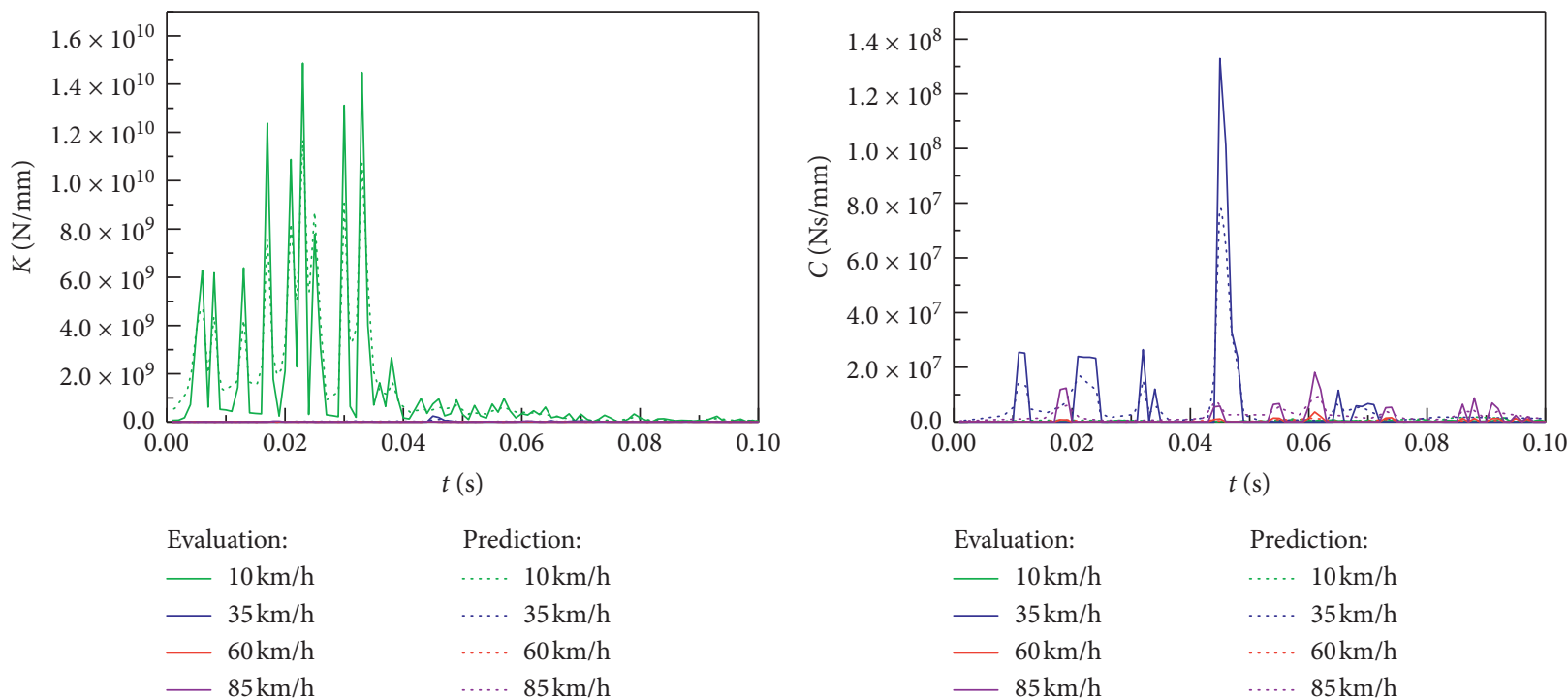

Figure 9: Coupler buffer device 3 comparisons of estimation and prediction.

displacement, velocity, and acceleration predicted by the CLGAN model, with the FE model results, as can be seen in Figure 14.

The figure shows the analysis of the results of both the CLGAN model and FE model. With regard to the FE model results, the maximum error of displacement is $7.6 \%$, the maximum error of velocity is $7.2 \%$, and the maximum error of acceleration is $29.6 \%$. The error is within the acceptable range, and the overall trend is consistent with the finite element results. For further verification, the dynamic characteristics of the anticlimbing device predicted by the CLGAN model method are also compared with the experimental results, as shown in Figure 15.

As can be seen in the figure, after the CLGAN model is compared with the FE model results, the maximum error of the displacement is $7.4 \%$, the maximum error of the velocity is $7.1 \%$, and the maximum error of the acceleration is $23.9 \%$. The error is again within the acceptable range, and the overall trend is consistent with the experimental results.

By comparing and analyzing these three representative methods (CLGAN model with test results and finite element analysis), the reliability and accuracy of the CLGAN model are verified from the perspective of dynamic characteristics: it can be seen that the trends of displacement and velocity are consistent; and even though the variable noise of acceleration is relatively large, possibly due to the sharp irregularities, the error is again within an acceptable range.

5.2. Head Car Model. In this section, the head car model is analyzed. Similar to before, the anticlimbing device's crash dynamic characteristics are simulated using the CLGAN 

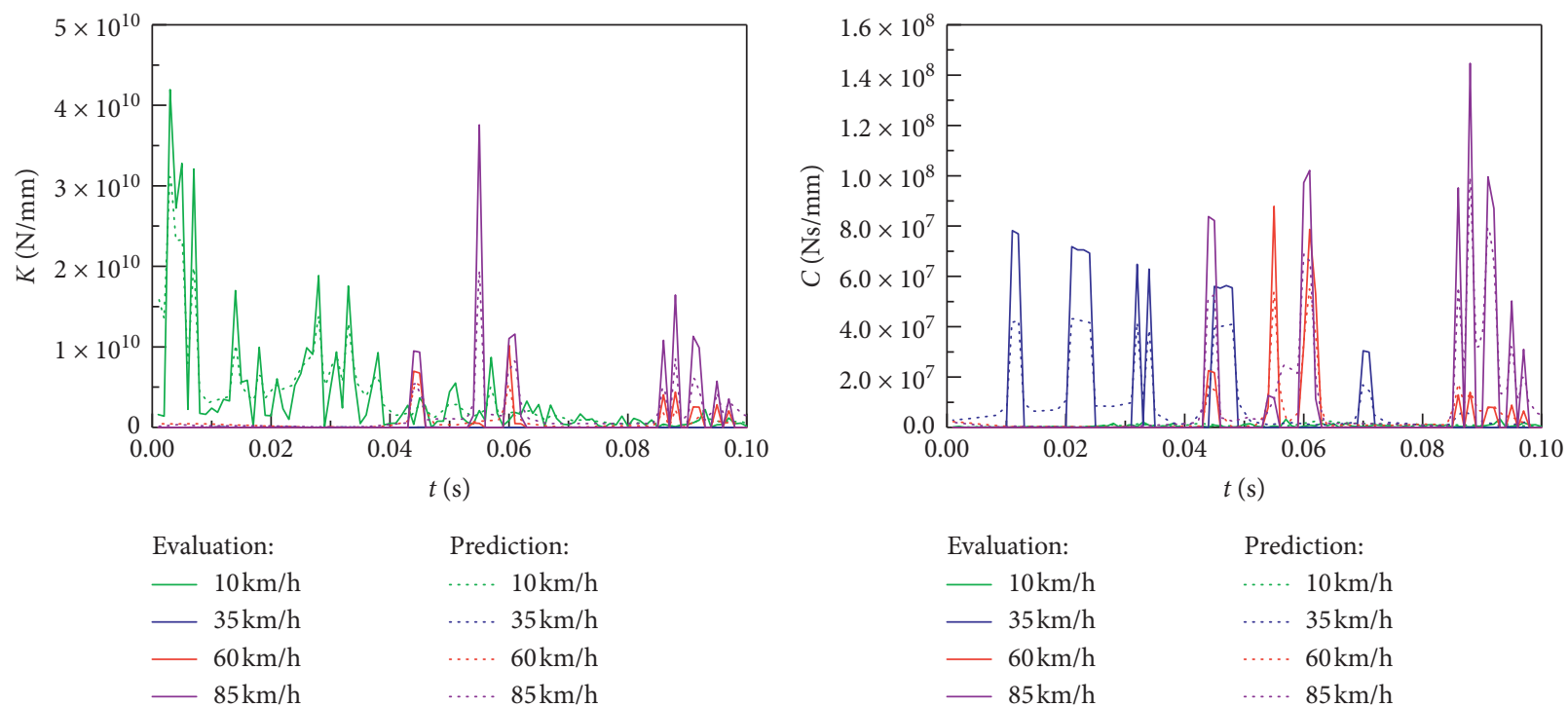

FIgURE 10: Coupler buffer device 4 comparisons of estimation and prediction.

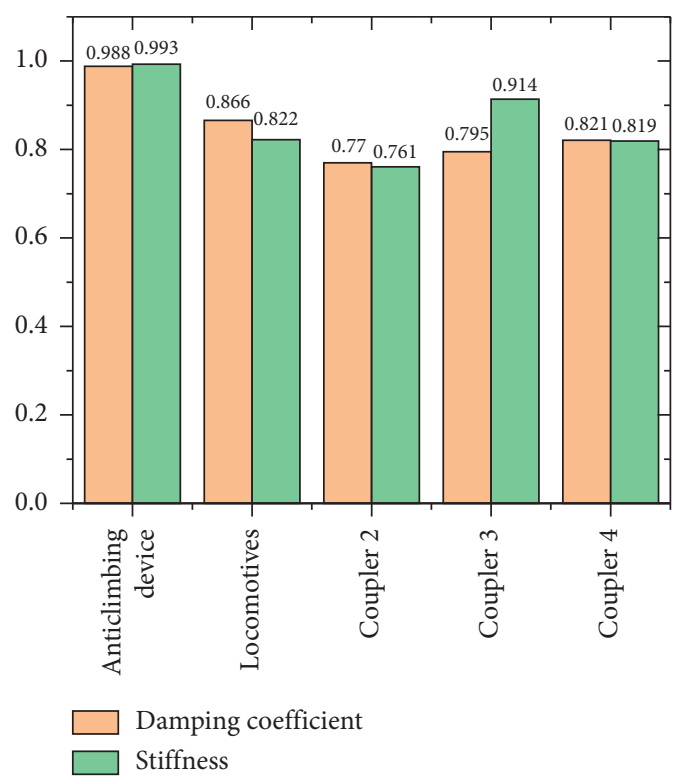

(a)

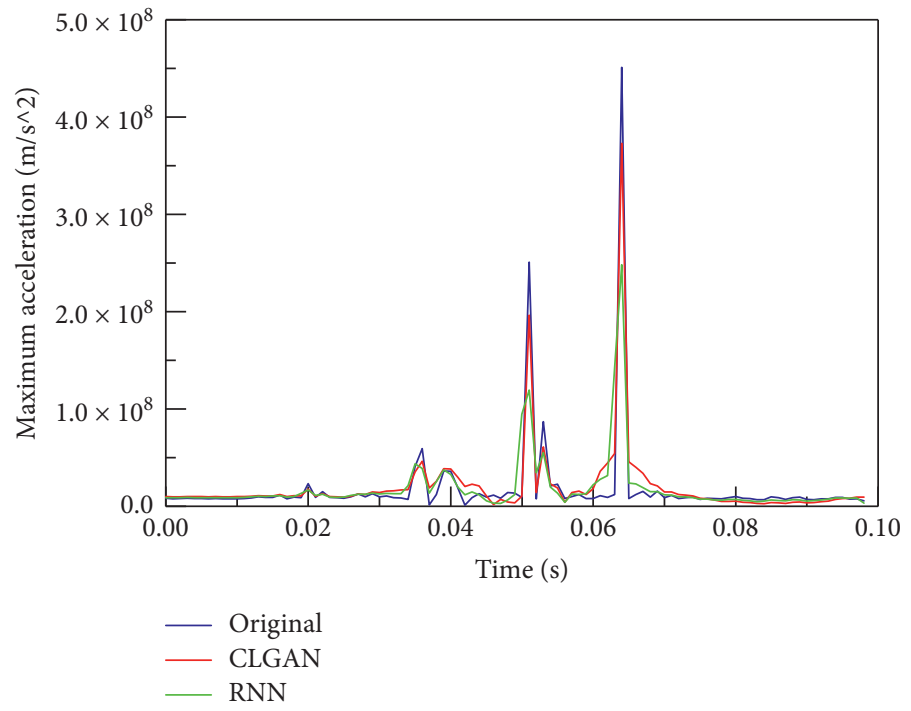

(b)

Figure 11: Estimation of CLGAN model. (a) Goodness of fit, $R^{2}$, of nonlinear parameters. (b) Comparison of different methods.

model and Newmark- $\beta$ method, and the results of the simulation are shown in Figure 16.

Again, the head car crash dynamic characteristics such as displacement, velocity, and acceleration predicted by the CLGAN model are compared with the FE model results, as shown in Figure 17.

As can be seen in the figure, after the CLGAN model is compared with the finite element results, the maximum error of the displacement is $5.8 \%$, the maximum error of the velocity is $9.1 \%$, and the maximum error of the acceleration is $29.2 \%$. The trends of displacement and velocity are consistent, thus confirming the effectiveness and accuracy of the CLGAN model from the perspective of dynamic characteristics.
5.3. Intermediate Car Model. In this section, the intermediate car model is analyzed: its crash dynamic characteristics are simulated using the CLGAN model and Newmark- $\beta$ method. The results of the simulation are shown in Figure 18.

Similar to the previous analysis, the intermediate car crash dynamic characteristics such as displacement, velocity, and acceleration predicted by the CLGAN model are compared with the FE model results, as shown in Figure 19.

In the figure, after comparing and analyzing the CLGAN model with the finite element results, we can see that the displacement, speed, and acceleration trends of the dynamic characteristics of the intermediate car essentially coincide with one another, and the error is within an acceptable 


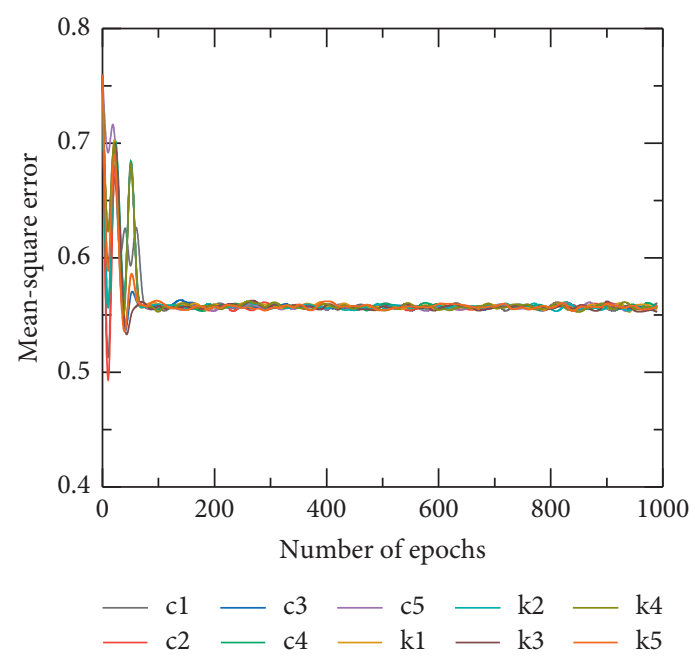

(a)

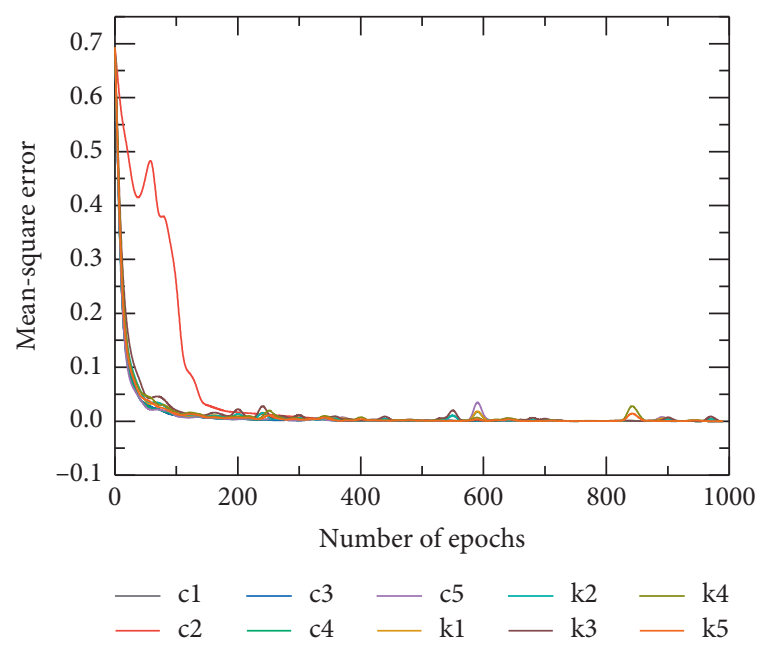

(b)

FIgure 12: Comparison chart of goodness of fit and loss curve. (a) Discriminator loss curve. (b) Adversarial generator loss curve.

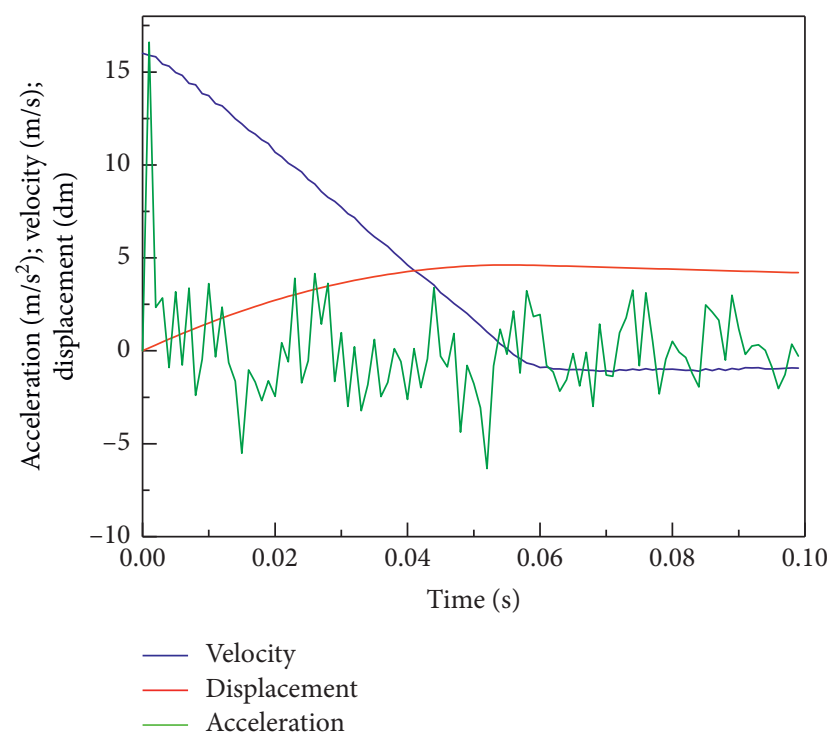

FIGURE 13: Results of the CLGAN model simulation.

range. Therefore, the reliability and accuracy of the CLGAN model are again verified from the perspective of the dynamic characteristics. From the comparison, we can also observe that the CLGAN model is able to make effective estimations for different key components. Although the acceleration plots do not exactly match, the overall shape of the estimated acceleration graph is preserved and corresponds to the reference measured acceleration. Similarity between the velocity and crush time histories was also achieved.
5.4. Modeling Comparison. A comparison of the FE analysis time and training time of the nonlinear lumped model applying the CLGAN neural network is reported in Table 2.

The results show that the CLGAN model demonstrates high accuracy in evaluating and predicting the nonlinear lumped model with nonlinear parameters, which is consistent with the analysis results of the FE modeling. Moreover, the time required is only one-tenth of what is 


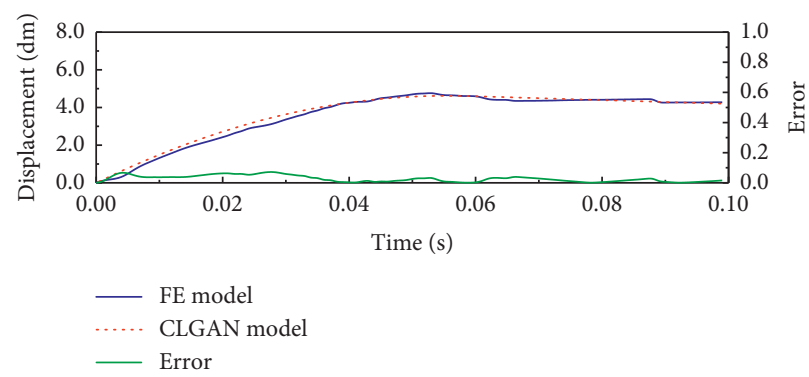

(a)
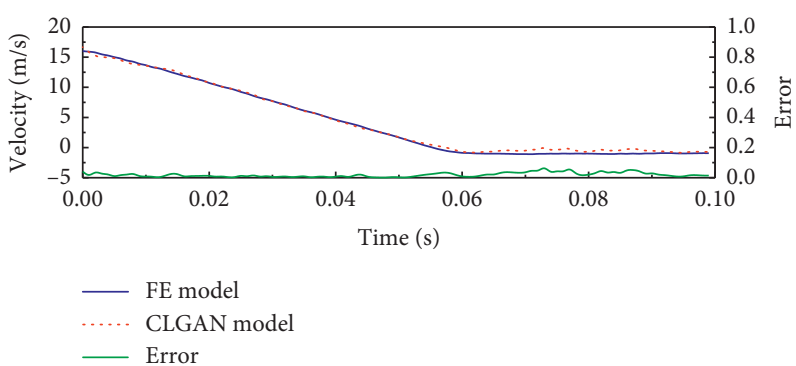

(b)

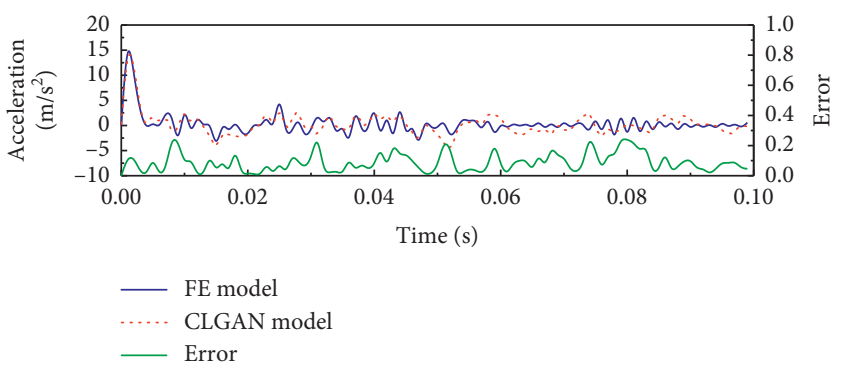

(c)

FIGURE 14: Comparison of the anticlimbing device's crash dynamic characteristics. (a) Displacement comparison of CLGAN model and FE model. (b) Velocity comparison of CLGAN model and FE model. (c) Acceleration comparison of CLGAN model and FE model.

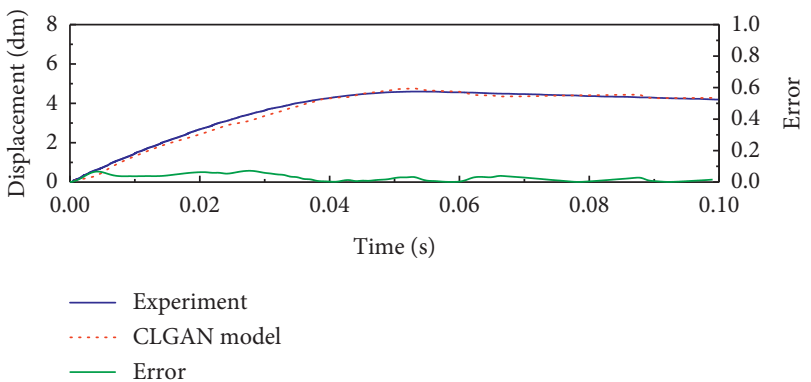

(a)

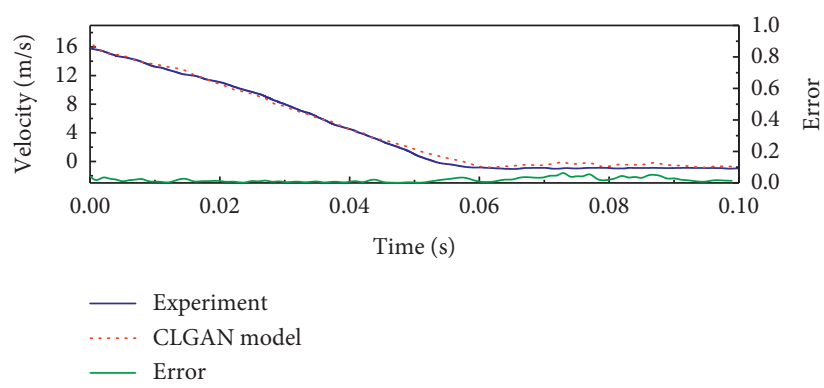

(b)

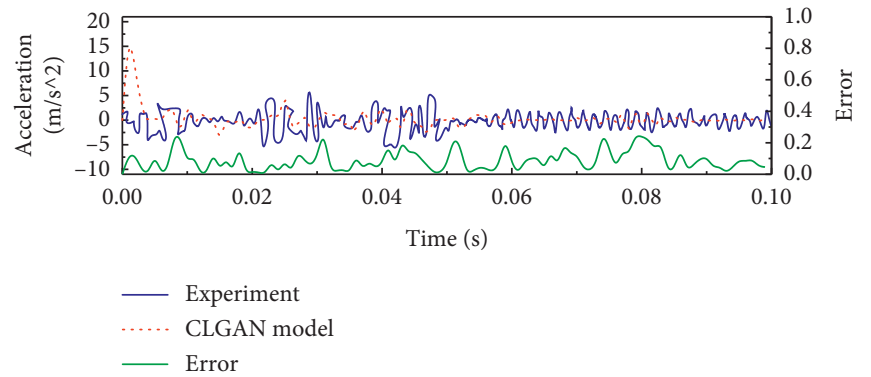

(c)

Figure 15: Comparison of the anticlimbing device's crash dynamic characteristics. (a) Displacement comparison of CLGAN model and experiment results. (b) Velocity comparison of CLGAN model and experiment results. (c) Acceleration comparison of CLGAN model and experiment results.

needed for the FE model. Therefore, the proposed model can achieve better estimation performance while having extremely low complexity, and it is also easy to establish with low time cost and economic.

\section{Application in Train Sets Crash Simulation}

In order to further evaluate the feasibility of the nonlinear lumped model in a train set crash simulation, we built 


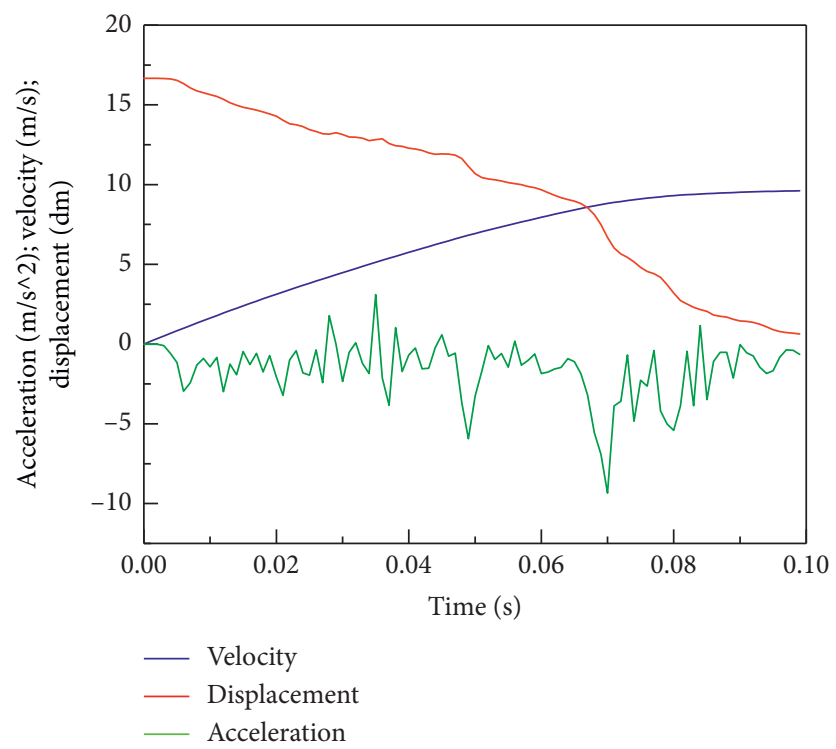

FIgURE 16: Results of the CLGAN model simulation.

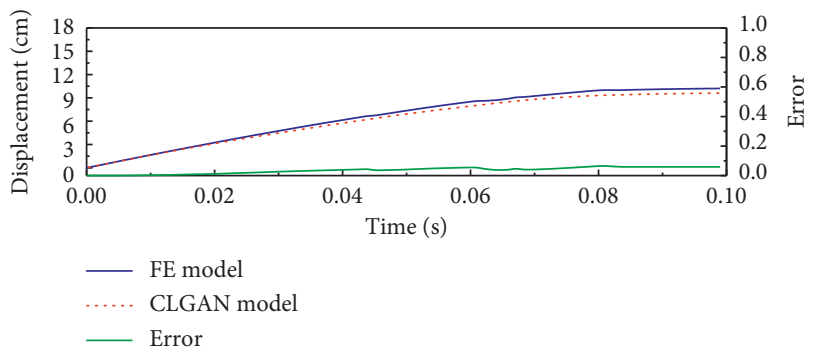

(a)

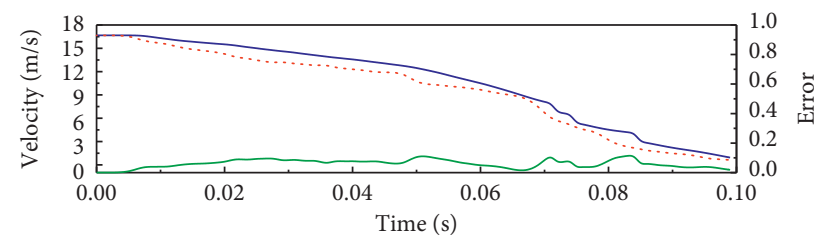

FE model

..... CLGAN model

Error

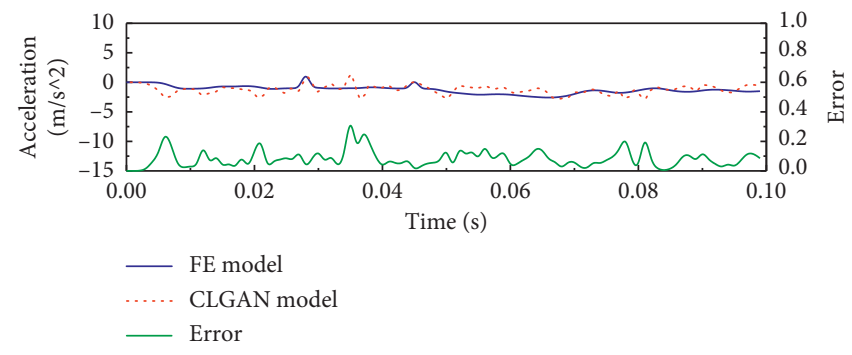

(c)

Figure 17: Comparison of the head car crash dynamic characteristics. (a) Displacement comparison of CLGAN model and FE model. (b) Velocity comparison of CLGAN model and FE model. (c) Acceleration comparison of CLGAN model and FE model.

dynamic models for the lead car and vehicles and connected them end to end to construct the dynamic model for a train set (see Figure 20), which can be used in train set crashworthiness research. In the simulation depicted in Figure 21, the left 4-vehicle train set, with assigned speed $V_{0}$, comes into crash with the right 4 -vehicle static train set.

In this case study, the initial velocity is set to $50 \mathrm{~km} / \mathrm{h}$. We build the nonlinear lumped model, where each vehicle corresponds to a rigid body whose end structures and couplers are substituted by nonlinear spring elements. The nonlinear parameters of the model at different initial speeds are predicted by applying the CLGAN neural network, and these are then substituted into the nonlinear lumped model. Then, the 4-vehicle train sets dynamic characteristics are predicted and evaluated, as shown in Figure 21.

Figure 21(a) shows that the crash displacement of the train increases with time. It can be observed that the displacement of the driving train increases directly after the crash, whereas, in the case of the static train, it increases gradually after the stationary stage mainly due to the fact that the latter has been buffered after crash. From Figure 21(b), 


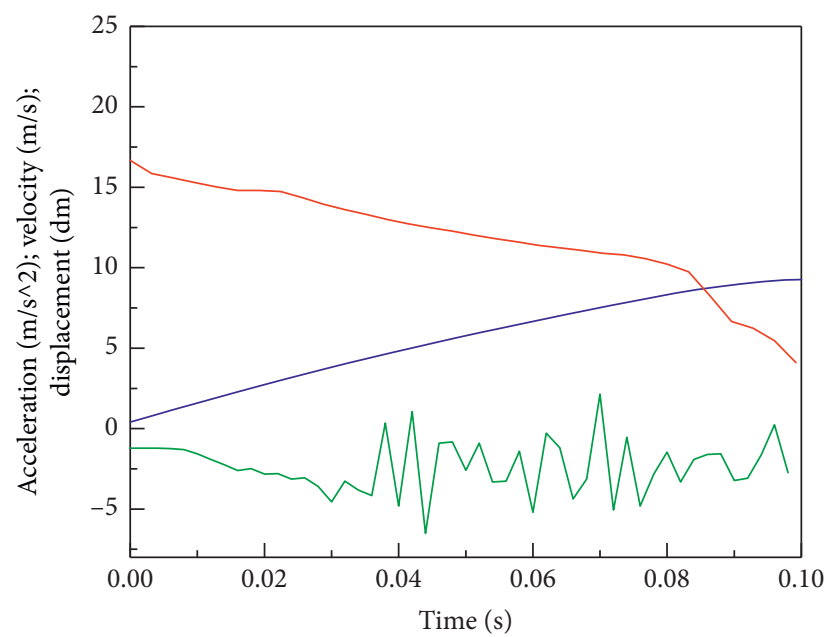

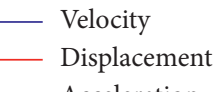

Figure 18: Results of the CLGAN model simulation.

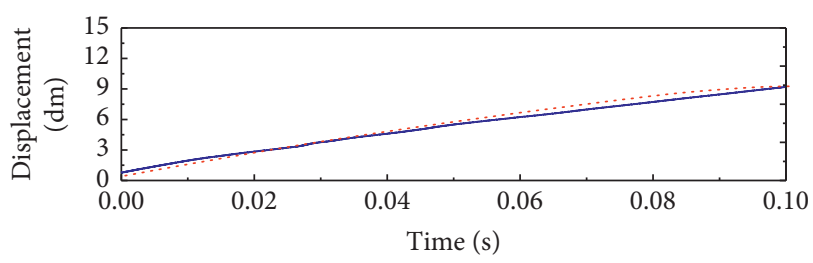

FE model

CLGAN model

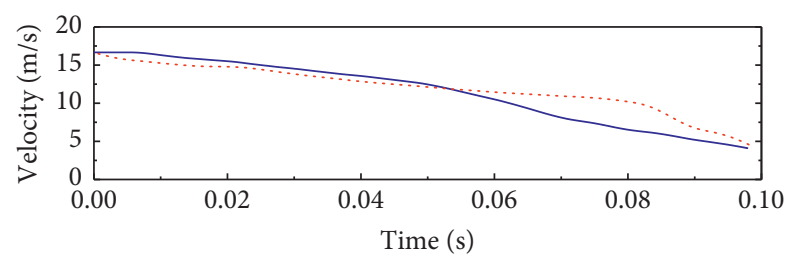

FE model

CLGAN model

(a)

(b)

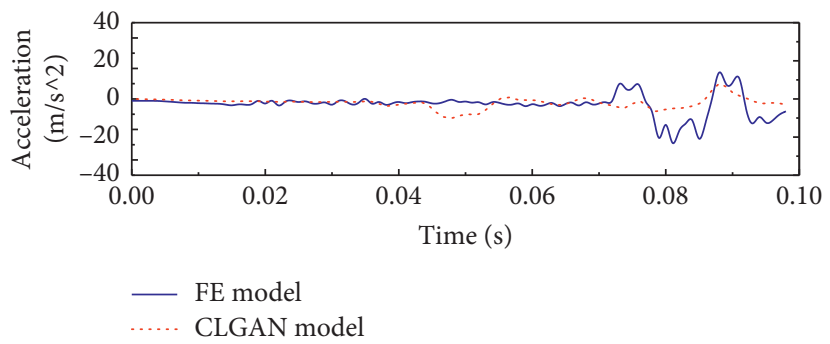

(c)

FIGURE 19: Comparison of crash dynamic characteristics obtained from different modeling. (a) Displacement comparison of CLGAN model and FE model. (b) Velocity comparison of CLGAN model and FE model. (c) Acceleration comparison of CLGAN model and FE model.

TABLE 2: The comparison of the analysis time of different models.

\begin{tabular}{lcccc}
\hline Model & Simulation time $(\mathrm{s})$ & Training time $(\mathrm{s})$ & Prediction time $(\mathrm{s})$ & Percentage $(\%)$ \\
\hline FE model & 426732 & - & - & - \\
Nonlinear lumped model & $32246.64+26927.96$ & $2216.47+961.77$ & $128.42+105.03$ & $13.8 \%$ \\
\hline
\end{tabular}

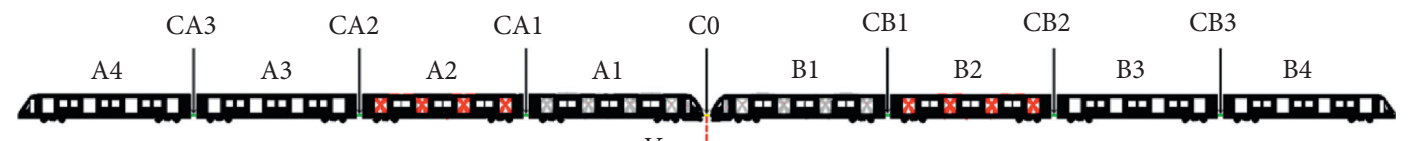

Driving train $V_{0}$

Static train

FIgURE 20: Head-on crash simulation between two 4-vehicle train sets. 


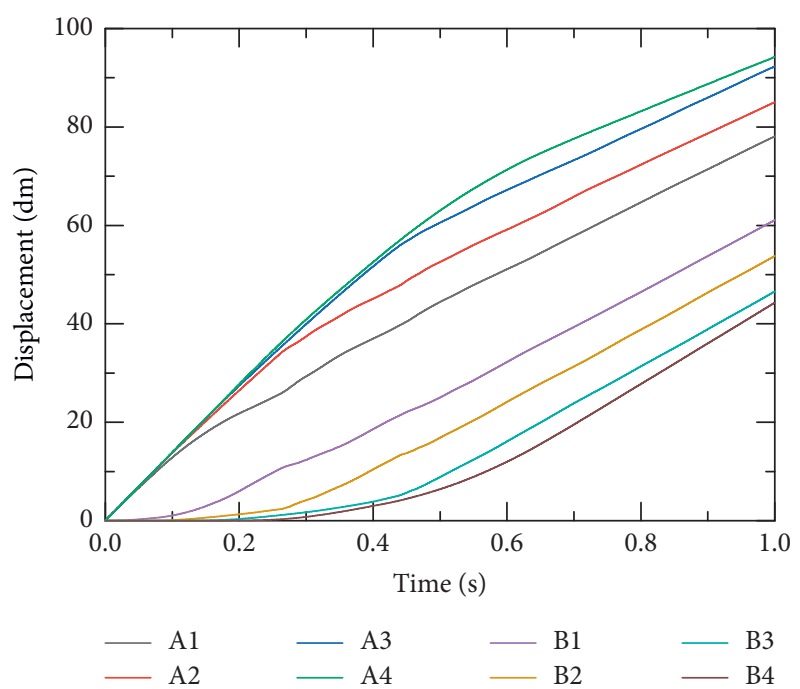

(a)

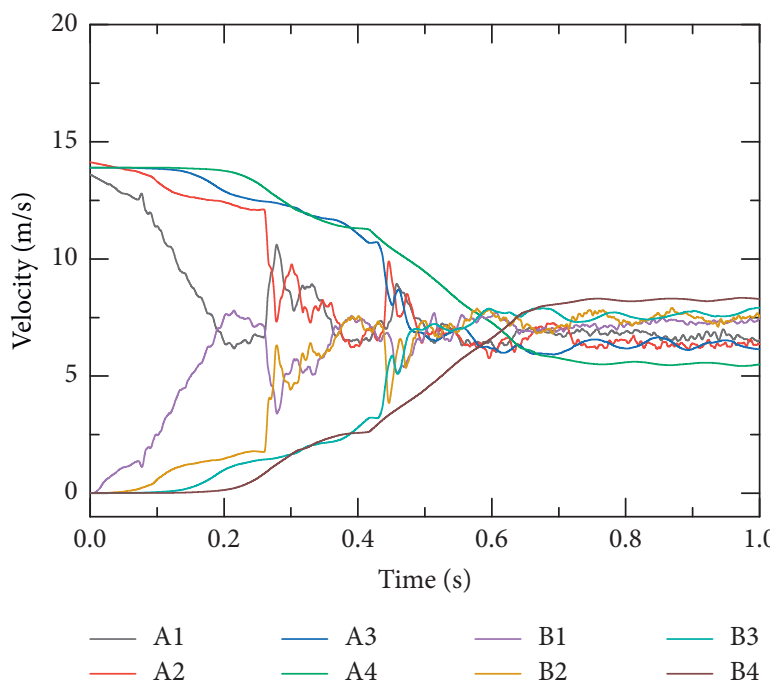

(b)

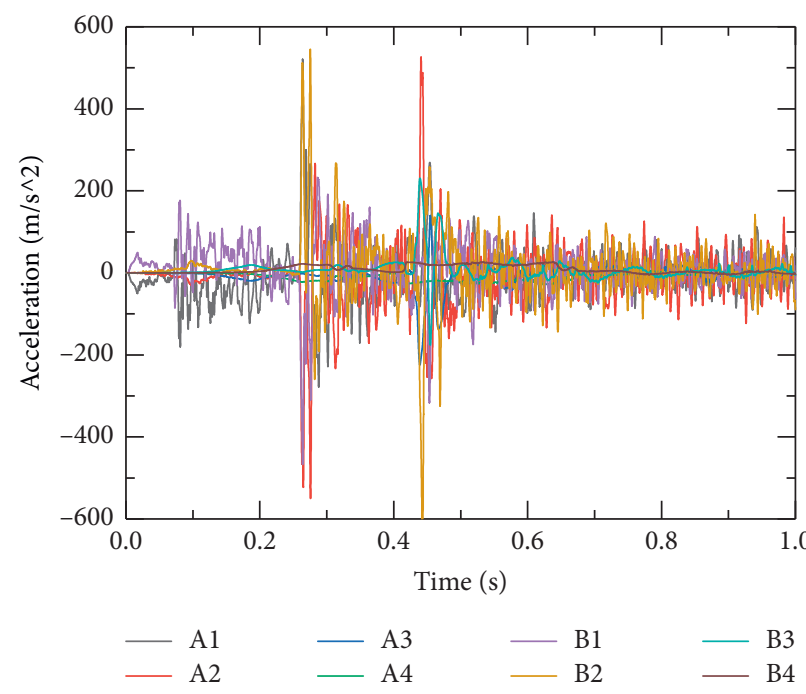

(c)

Figure 21: Dynamic characteristics of each vehicle in 4-vehicle train sets crash. (a) Displacement characteristics. (b) Velocity plots. (c) Acceleration signals.

the graph of the velocity of the active train shows a downward trend during the crash process; meanwhile the static train begins speeding up. At $0.6 \mathrm{~s}$, the two vehicles reach the same velocity: from this phenomenon, we can infer that the velocities of the middle vehicles in the two train sets have two stages, which correspond to the different velocities when exerting force from couplers and end structures, respectively. Finally, from Figure 21(c), it can be observed that the acceleration curves have multiple peak points, which means that the acceleration decreases with some timevarying fluctuations rather than in a strictly monotonic manner. This is mainly because each vehicle in the two train sets collides with the neighboring vehicle.

\section{Conclusions}

This work is essentially based on the inherent relationship between the nonlinear parameters and the dynamic characteristics of the frontal crash process of the Beijing metro. Nonlinearity in the system derives from a huge number of elements, joints, and connections involved in a crash event. Thus, a nonlinear lumped model that applies the CLGAN model is proposed, which shows a good correspondence with the deformation process in the experimental results by evaluating and observing ten time-varying nonlinear parameters. Compared to previous crash models, the proposed method describes the kinetic signal of a model directly and includes the 
deformation features of the vehicle structure. This enables the model to balance its accuracy and complexity properly.

An integrated crash estimation scheme is also proposed in this work: for each type of crash, the structure of its signals is firstly given, and then the parameters are, respectively, investigated according to the velocity change. Our case studies give examples of the application of the proposed scheme and demonstrate its effectiveness. In addition, it provides a new way of thinking for engineers when it comes to optimizing the performance of the anticlimbing energy absorber and coupler buffer devices. Compared to traditional modeling methods, the main contributions of this work are summarized as follows:

(1) The combination of the nonlinear spring-massdamper modeling with Deep Learning is a new technology that can potentially have a higher ability in estimating and predicting dynamic characteristics while maintaining lower complexity.

(2) The CLGAN model is proposed to explore the nonlinearity of the stiffness and damping coefficients, and this reveals the mathematical expressions between the structural parameters and the dynamic characteristics of the system. The CLGAN model shows great potential in nonlinear estimation, and it can better describe the variation of nonlinear spring damping compared with RNN model.

(3) The obtained results have a significant impact, especially in the early concept design stages when a detailed finite element model is usually not available. Moreover, this model is easy to build, economical, and practical and has high computational efficiency.

(4) The model can accurately evaluate and reproduce the variation of the nonlinear parameters of the key components during the train crash process.

A limitation of our approach is that the present method has restricted applicability to a specific type of train. In the future, it will be interesting to investigate the performance of another estimation algorithm, as there exists a variety of different approaches that can be utilized for this task. Finally, we believe this work has the potential to benefit the design of other safety functions, and further studies on the dynamics of crashes occurring under more complex conditions (e.g., oblique and offset cases) should be carried out in the future.

\section{Data Availability}

The curve data used to support the findings of this study are available from the corresponding author upon request.

\section{Conflicts of Interest}

The authors declare that they have no conflicts of interest.

\section{Acknowledgments}

This work was supported by the National Key Research and Development Project (2019YFB1405401) and the Research
Funds for the National Natural Science Foundation of China (51405402). The financial support is gratefully acknowledged.

\section{References}

[1] H. Dong, B. Ning, B. Cai, and Z. Hou, "Automatic train control system development and simulation for high-speed railways," IEEE Circuits and Systems Magazine, vol. 10, no. 2, pp. 6-18, 2010.

[2] J.-W. Sheu and W.-S. Lin, "Energy-saving automatic train regulation using dual heuristic programming," IEEE Transactions on Vehicular Technology, vol. 61, no. 4, pp. 1503-1514, 2012.

[3] J. Yin, D. Chen, and L. Li, "Intelligent train operation algorithms for subway by expert system and reinforcement learning," IEEE Transactions on Intelligent Transportation Systems, vol. 15, no. 6, pp. 2561-2571, 2014.

[4] D. Ibrahim, "Smart train collision detection system using a microcontroller," International Journal of Computer Applications, vol. 152, no. 2, pp. 16-22, 2016.

[5] B. B. Munyazikwiye, H. R. Karimi, and K. G. Robbersmyr, "A mathematical model for vehicle-occupant frontal crash using genetic algorithm," in Proceedings of the 2016 UKSim-AMSS 18th International Conference on Computer Modelling and Simulation (UKSim), IEEE, Cambridge, UK, April 2016.

[6] Y. Dai and C. Duan, "Beam element modelling of vehicle body-in-white applying artificial neural network," Applied Mathematical Modeling, vol. 33, no. 3, pp. 2808-2817, 2009.

[7] H. R. Karimi, W. Pawlus, and K. G. Robbersmyr, "A recursive model for nonlinear spring-mass-damper estimation of a vehicle localized impact," in Proceedings of the 4th WSEAS International Conference on Energy and Development-Environment-Biomedicine, pp. 473-478, Stevens Point, WI, USA, July 2011.

[8] X. Zhou, L. Cao, I. Khan, and Q. Li, "Data inspecting and denoising method for data-driven stochastic subspace identification," Shock and Vibration, vol. 2018, Article ID 3926817 , 11 pages, 2018.

[9] P. L. N. U. Cooray and T. D. Rupasinghe, "Machine learningbased parameter tuned genetic algorithm for energy minimizing vehicle routing problem," Journal of Industrial Engineering, vol. 2017, Article ID 3019523, 13 pages, 2017.

[10] Q. Xue, K. Wang, J. J. Lu, and Y. Liu, "Rapid driving style recognition in car-following using machine learning and vehicle trajectory data," Journal of Advanced Transportation, vol. 2019, Article ID 9085238, 11 pages, 2019.

[11] M. Stoffel, F. Bamer, and B. Markert, "Artificial neural networks and intelligent finite elements in non-linear structural mechanics," Thin-Walled Structures, vol. 131, pp. 102-106, 2018.

[12] W. Pawlus, H. R. Karimi, and K. G. Robbersmyr, "Reconstruction and simulation of the vehicle to road safety barrier oblique collision based on the Levenberg-Marquardt algorithm," International Journal of Crashworthiness, vol. 17, no. 6, pp. 676-692, 2012.

[13] W. Pawlus, H. R. Karimi, and K. G. Robbersmyr, "Data-based modeling of vehicle collisions by nonlinear autoregressive model and feedforward neural network," Information Sciences, vol. 235, pp. 65-79, 2013.

[14] B. B. Munyazikwiye, D. Vysochinskiy, M. Khadyko et al., "Prediction of vehicle crashworthiness parameters using piecewise lumped parameters and finite element models," Designs, vol. 2, no. 43, pp. 2-16, 2018.

[15] Z. Tang, Y. Zhu, Y. Nie et al., "Data-driven train set crash dynamics simulation," Vehicle System Dynamics, vol. 55, no. 2, pp. 149-167, 2017. 
[16] E. Acar, "Increasing automobile crash response metamodel accuracy through adjusted cross validation error based on outlier analysis," International Journal of Crashworthiness, vol. 20, no. 2, pp. 107-122, 2015.

[17] Z. Wei, K. G. Robbersmyr, and H. R. Karimi, "Data-based modeling and estimation of vehicle crash processes in frontal fixed-barrier crashes," Journal of the Franklin Institute, vol. 354, no. 12, pp. 4896-4912, 2017.

[18] L. Zhao, W. Pawlus, H. R. Karimi, and K. G. Robbersmyr, "Data-based modeling of vehicle crash using adaptive neuralfuzzy inference system," IEEE/ASME Transactions on Mechatronics, vol. 19, no. 2, pp. 684-696, 2014.

[19] B. B. Munyazikwiye, H. R. Karimi, and K. G. Robbersmyr, "Fuzzy logic approach to predict vehicle crash severity from acceleration data," in Proceedings of the 2015 International Conference on Fuzzy Theory and its Applications, pp. 18-20, IEEE, Yilan, Taiwan, November 2015.

[20] H. R. Karimi, W. Pawlus, K. G. Robbersmyr, and K. G. Robbersmyr, "Signal reconstruction, modeling and simulation of a vehicle full-scale crash test based on Morlet wavelets," Neurocomputing, vol. 93, pp. 88-99, 2012.

[21] T. Zhu, S. Liu, S.-n Xiao et al., "Train crash dynamic model considering longitudinal and vertical coupling," Advances in Mechanical Engineering, vol. 11, no. 1, pp. 168-781, 2019.

[22] R. A. Oprea, "A constrained motion perspective of railway vehicles collision," Multibody System Dynamics, vol. 30, no. 1, pp. 101-116, 2013.

[23] P. F. Carlbom, "Combining MBS with FEM for rail vehicle dynamics analysis," Multibody System Dynamics, vol. 6, no. 3, pp. 291-300, 2001.

[24] B. Vujanovic, "Conservation laws of dynamical systems via d'alembert's principle," International Journal of Non-linear Mechanics, vol. 13, no. 3, pp. 185-197, 1978.

[25] A. El Kacimi, P. K. Woodward, O. Laghrouche, and G. Medero, "Time domain 3D finite element modelling of train-induced vibration at high speed," Computers \& Structures, vol. 118, pp. 66-73, 2013.

[26] P. T. Nguyen, T. D. Pham, and H. P. Hoang, "A nonlinear dynamic foundation model for dynamic response of tracktrain interaction," Shock and Vibration, vol. 2020, pp. 1-10, 2020.

[27] F. Pukelsheim, "The three sigma rule," The American Statistician, vol. 48, no. 2, pp. 88-91, 1994.

[28] S. K. Turitsyn, J. E. Prilepsky, S. T. Le et al., "Nonlinear Fourier transform for optical data processing and transmission: advances and perspectives," Optica, vol. 4, no. 3, p. 307, 2017.

[29] P. S. Addison, The Illustrated Wavelet Transform Handbook: Introductory Theory and Applications in Science, Engineering, Medicine and Finance, CRC Press, Boca Raton, FL, USA, 2017.

[30] J. P. Amezquita-Sanchez, H. S. Park, and H. Adeli, "A novel methodology for modal parameters identification of large smart structures using MUSIC, empirical wavelet transform, and Hilbert transform," Engineering Structures, vol. 147, pp. 148-159, 2017.

[31] S. Huang, J. Tang, J. Dai, Y. Wang, and J. Dong, "1DCNN fault diagnosis based on cubic spline interpolation pooling," Shock and Vibration, vol. 2020, Article ID 1949863, 13 pages, 2020.

[32] Z. Zhao, W. Chen, X. Wu et al., "LSTM network: a deep learning approach for short-term traffic forecast," IET Intelligent Transport Systems, vol. 11, pp. 68-75, 2017.

[33] S. Pascual, A. Bonafonte, and J. Serra, "Speech enhancement generative adversarial network," https://arxiv.org/abs/1703. 09452.
[34] I. Goodfellow, J. Pouget-Abadie, M. Mirza et al., "Generative adversarial nets," Advances in Neural Information Processing Systems, pp. 2672-2680, ACM, New York, NY, USA, 2014.

[35] O. Heirich, A. Lehner, P. Robertson et al., "Measurement and analysis of train motion and railway track characteristics with inertial sensors," in Proceedings of the 2011 14th International IEEE Conference on Intelligent Transportation Systems (ITSC), IEEE, Washington, DC, USA, October 2011.

[36] X. Xue, F. Schmid, and R. A. Smith, "Analysis of the structural characteristics of an intermediate rail vehicle and their effect on vehicle crash performance," Proceedings of the Institution of Mechanical Engineers, Part F: Journal of Rail and Rapid Transit, vol. 221, no. 3, pp. 339-352, 2007.

[37] S. Xie and H. Zhou, "Impact characteristics of a composite energy absorbing bearing structure for railway vehicles," Composites Part B: Engineering, vol. 67, pp. 455-463, 2014.

[38] S. Y. Chang, "Studies of Newmark method for solving nonlinear systems: (I) basic analysis," Journal of the Chinese Institute of Engineers, vol. 27, no. 5, pp. 651-662, 2004. 